\title{
Individual kinetochore-fibers locally dissipate force to maintain robust mammalian spindle structure
}

\author{
Alexandra F. Long ${ }^{1,3,4}$ (D), Pooja Suresh ${ }^{2,3,4}$ (D), and Sophie Dumont ${ }^{1,2,3,4,5}$ (D)
}

\begin{abstract}
At cell division, the mammalian kinetochore binds many spindle microtubules that make up the kinetochore-fiber. To segregate chromosomes, the kinetochore-fiber must be dynamic and generate and respond to force. Yet, how it remodels under force remains poorly understood. Kinetochore-fibers cannot be reconstituted in vitro, and exerting controlled forces in vivo remains challenging. Here, we use microneedles to pull on mammalian kinetochore-fibers and probe how sustained force regulates their dynamics and structure. We show that force lengthens kinetochore-fibers by persistently favoring plus-end polymerization, not by increasing polymerization rate. We demonstrate that force suppresses depolymerization at both plus and minus ends, rather than sliding microtubules within the kinetochore-fiber. Finally, we observe that kinetochore-fibers break but do not detach from kinetochores or poles. Together, this work suggests an engineering principle for spindle structural homeostasis: different physical mechanisms of local force dissipation by the k-fiber limit force transmission to preserve robust spindle structure. These findings may inform how other dynamic, force-generating cellular machines achieve mechanical robustness.
\end{abstract}

\section{Introduction}

The spindle segregates chromosomes at cell division and must do so accurately and robustly for proper cell and tissue function. In mammalian spindles, bundles of 15-25 microtubules called kinetochore-fibers (k-fibers) span from the kinetochore at their plus ends to the spindle pole at their minus ends (Rieder, 1981; McDonald et al., 1992; McEwen et al., 1997). The k-fibers are dynamic at both ends (Mitchison, 1989; Cassimeris and Salmon, 1991), and we now have a wealth of information on the molecular regulation of their dynamics (Cheeseman and Desai, 2008; Bakhoum and Compton, 2012; Monda and Cheeseman, 2018). To move chromosomes, $\mathrm{k}$-fibers generate force through plus-end depolymerization (Mitchison et al., 1986; Koshland et al., 1988; Grishchuk et al., 2005). Yet, while we are beginning to understand how the mammalian k-fiber generates force (Inoué and Salmon, 1995; Grishchuk, 2017), we know much less about how force from the k-fiber and surrounding spindle in turn affects k-fiber structure and dynamics. Defining this relationship between k-fibers and their mechanical environment is central to understanding spindle structural homeostasis and function.

Force affects microtubule dynamics and structure in a variety of contexts (Dogterom et al., 2005). From in vitro experiments coupling single microtubules to yeast kinetochore protein complexes, we know that force can regulate all four parameters of microtubule dynamic instability (Franck et al., 2007; Akiyoshi et al., 2010; Sarangapani et al., 2013): it increases polymerization rates while slowing depolymerization, and it favors rescue over catastrophe. From in vivo experiments, we know that force exerted by the cell correlates with changes in k-fiber dynamics (Rieder et al., 1986; Skibbens et al., 1993; Wan et al., 2012; Dumont et al., 2012; Auckland et al., 2017) and that reducing and increasing force can bias $\mathrm{k}$-fiber dynamics in different systems (Nicklas and Staehly, 1967; Skibbens et al., 1995; Khodjakov and Rieder, 1996; Skibbens and Salmon, 1997). However, the feedback between force, structure, and dynamics in the mammalian k-fiber remains poorly understood. For example, we do not know which dynamic instability parameters are regulated by force, or at which microtubule end. Similarly, we do not know how microtubules within the $\mathrm{k}$-fiber remodel their structure (e.g., slide or break) under force, or the physical limits of the connections between k-fibers and the spindle. These questions are at the heart of understanding how the spindle can maintain its structure given its dynamic, force-generating parts (Oriola et al., 2018; Elting et al., 2018). Addressing these questions requires the ability to apply force on k-fibers with spatial and

${ }^{1}$ Tetrad Graduate Program, University of California, San Francisco, San Francisco, CA; ${ }^{2}$ Biophysics Graduate Program, University of California, San Francisco, San Francisco, CA; ${ }^{3}$ Department of Cell and Tissue Biology, University of California, San Francisco, San Francisco, CA; ${ }^{4}$ Department of Bioengineering and Therapeutic Science, University of California, San Francisco, San Francisco, CA; ${ }^{5}$ Chan Zuckerberg Biohub, San Francisco, CA.

Correspondence to Sophie Dumont: sophie.dumont@ucsf.edu; Alexandra F. Long: a.fitz.long@gmail.com.

(c) 2020 Long et al. This article is distributed under the terms of an Attribution-Noncommercial-Share Alike-No Mirror Sites license for the first six months after the publication date (see http://www.rupress.org/terms/). After six months it is available under a Creative Commons License (Attribution-Noncommercial-Share Alike 4.0 International license, as described at https://creativecommons.org/licenses/by-nc-sa/4.0/). 
temporal control, while concurrently imaging their dynamics. Yet, exerting controlled forces in dividing mammalian cells remains a challenge, and mammalian spindles and $\mathrm{k}$-fibers cannot currently be reconstituted in vitro. Chemical and genetic perturbations can change forces on $\mathrm{k}$-fibers in vivo, but these alter microtubule structure or dynamics, either directly or indirectly through regulatory proteins (De Brabander et al., 1986; Jaqaman et al., 2010; Alushin et al., 2014). Thus, direct mechanical approaches are needed inside mammalian cells.

Here, we use glass microneedles to directly exert force on individual $\mathrm{k}$-fibers inside mammalian cells and determine how their structure and dynamics remodel under sustained force. Inspired by experiments in insect spermatocytes (Nicklas and Staehly, 1967; Nicklas, 1997; Lin et al., 2018), we sought to adapt microneedle manipulation to pull on k-fibers in mitotic mammalian cells for many minutes while monitoring their dynamics with fluorescence imaging. We show that forces applied for minutes regulate $\mathrm{k}$-fiber dynamics at both ends, causing $\mathrm{k}$-fiber lengthening, but do not cause sliding of the microtubules within them. Furthermore, we demonstrate that sustained forces can break k-fibers rather than detach them from kinetochores or poles. Thus, k-fibers respond as a coordinated mechanical unit by remodeling at different sites to locally dissipate force, while preserving the connections between chromosomes and the spindle. Together, these findings suggest local force dissipation as an engineering principle for the dynamic spindle to maintain its structure and function under force and for other cellular machines to do the same.

\section{Results and discussion}

Microneedle manipulation of mammalian spindles enables sustained force application on $\mathbf{k}$-fibers with spatial and temporal control

To determine how mammalian k-fibers remodel under force, we sought an approach to apply forces with spatial and temporal control for sustained periods, compatible with cell health and live imaging of structure and dynamics. We adapted microneedle manipulation to pull on individual k-fibers in mammalian cells (Fig. $1 \mathrm{~A}$ ) and developed methods to do so gently enough to exert force for several minutes (Suresh et al., 2020). We used PtK cells as these cells are large, flat, molecularly tractable (Udy et al., 2015), and have few chromosomes so we can pull on individual $\mathrm{k}$-fibers. We used a micromanipulator and a fluorescently labeled glass microneedle to contact a target metaphase PtK cell. We inserted the microneedle vertically into the spindle, placing its tip deeper in the cell than the k-fiber to be manipulated. The needles we used had a $1.2 \pm 0.1 \mu \mathrm{m}$ shaft diameter in the focal plane of the manipulated k-fiber. Pulling on an outermost $k$-fiber in the spindle for several minutes, we could reproducibly exert controlled forces, moving the microneedle with specific velocities over any given duration (Fig. 1 B) and direction. The microneedle only locally deformed the cell membrane and spindle and remained outside of the cell, allowing precise, local control of where force is applied (Fig. $1 \mathrm{C}$; Suresh et al., 2020). Upon careful removal of the microneedle, we observed cells enter anaphase (Fig. 1 D), although in most
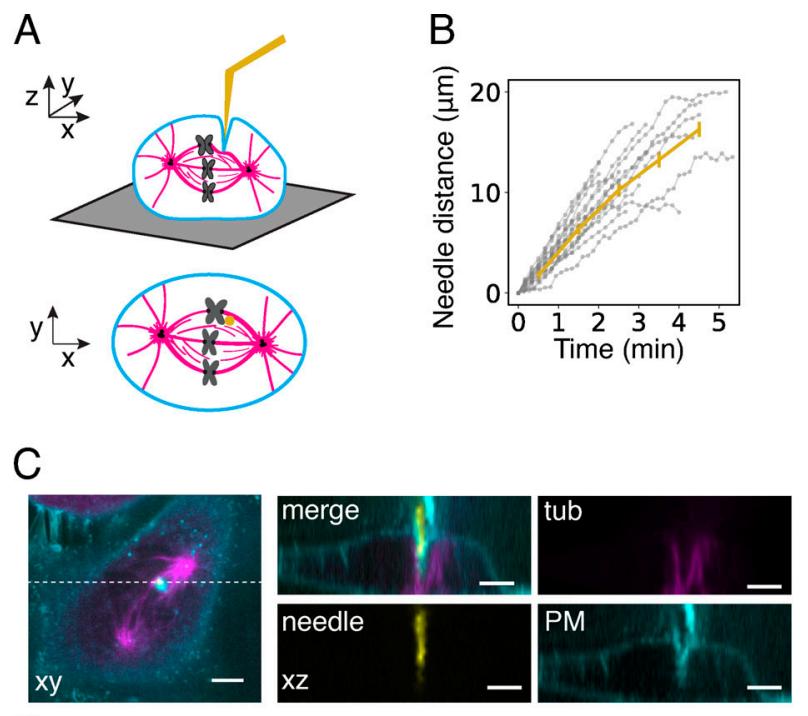

D

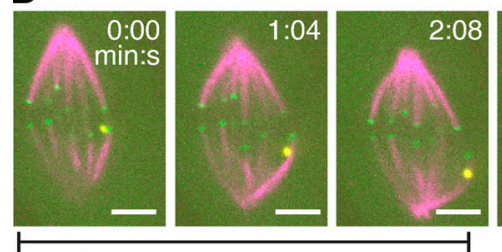

Microneedle manipulation

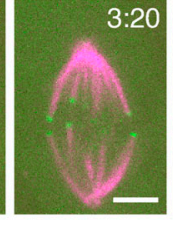

Recovery

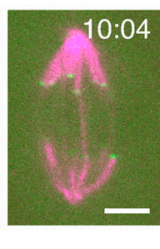

Anaphase
Figure 1. Microneedle manipulation of mammalian spindles enables sustained force application on k-fibers with spatial and temporal control. (A) Cartoon representation of microneedle (yellow) placement (3D and cross section) in a metaphase mammalian cell to exert sustained force on a k-fiber. (B) Plot of linear microneedle displacement over time during manipulation in metaphase PtK cell (mean \pm SEM, $n=18$ cells). This approach allows smooth, reproducible pulls on single mammalian k-fibers. (C) Representative z-stack reconstruction shows geometry of microneedle contact with the cell and metaphase spindle (tub; GFP-tubulin, magenta) as diagrammed in (A). The plasma membrane (PM; CellMask Orange dye, cyan) locally deforms around the microneedle (Alexa 647, yellow), but does not alter whole cell shape or puncture the cell. Scale bar, $4 \mu \mathrm{m}$. (D) Representative time-lapse images of microneedle (Alexa 555, yellow) manipulation to exert force on a k-fiber: it displaces the metaphase spindle (Cdc20-YFP, green; SiR-tubulin, magenta) and deforms the pulled k-fiber. Manipulated spindles can progress to anaphase (here at 10:04). Scale bar, $4 \mu \mathrm{m}$. See also Video 1.

cells the spindle could not be followed after needle removal (see Materials and methods). These observations are consistent with cell health maintenance during these sustained manipulations. Thus, we can use microneedle manipulation to exert forces with spatial and temporal control over minutes on a mammalian $\mathrm{k}$-fiber and thereby probe how force regulates k-fiber structure and dynamics.

\section{Individual mammalian k-fibers switch to persistent lengthening in response to sustained applied force}

To probe the response of $\mathrm{k}$-fibers to force, we placed the microneedle along the k-fiber, within a few micrometers of the outermost sister kinetochore pair (Fig. 2, A and B, and Fig. S1, A and B). We moved the microneedle at a speed of $5.2 \pm 0.2 \mu \mathrm{m} /$ min for $3.1 \pm 0.3$ min (Fig. $1 \mathrm{~B}$ ), perpendicular to the spindle's long axis at the start of manipulation. We predicted that in 


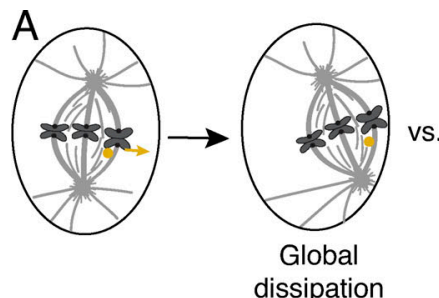

C

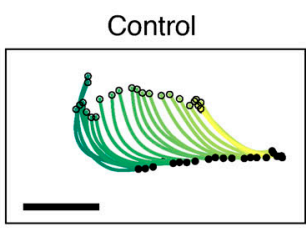

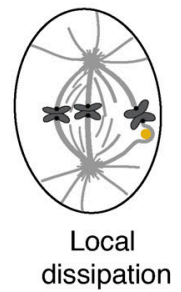

dissipation
B

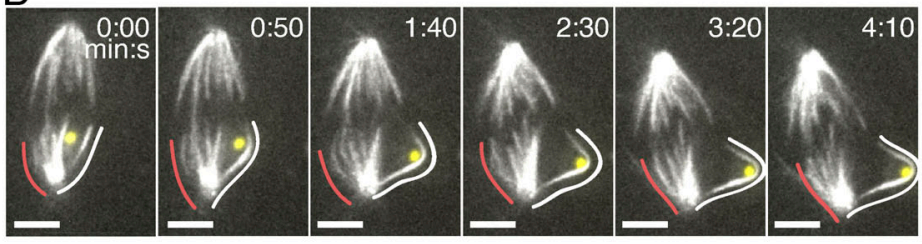

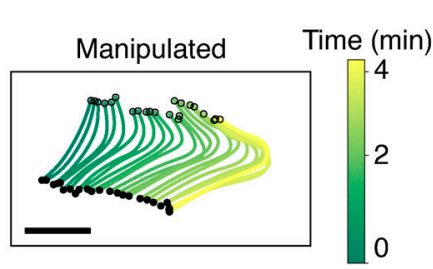
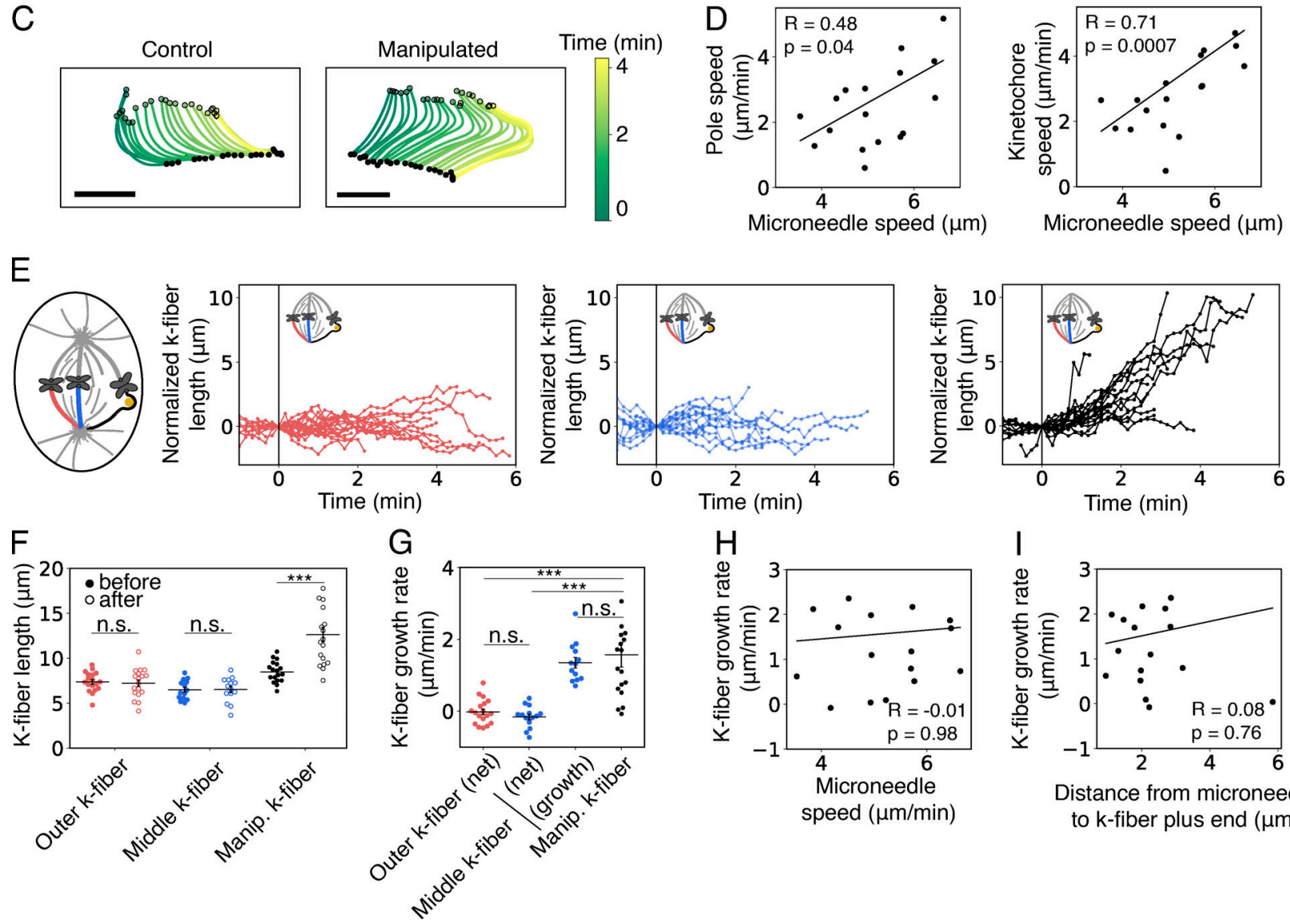

$\mathrm{H}$
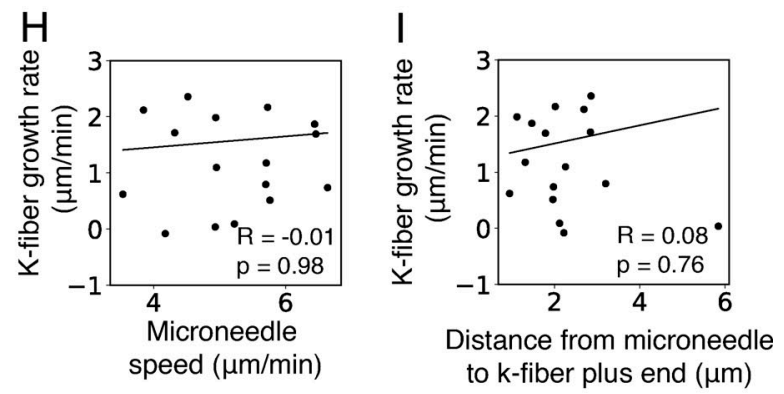

Figure 2. Individual mammalian $\mathbf{k}$-fibers switch to persistent lengthening in response to sustained applied force. (A) Assay to locally exert force on an outer k-fiber using a microneedle (yellow circle) to probe its response to force (yellow arrow). Possible outcomes include global movement of the whole spindle and local deformation of the k-fiber, reflecting global and local dissipation of applied force, respectively. vs., versus. (B) Representative time-lapse images of spindle and k-fiber (SiR-tubulin, white) movement and remodeling in response to sustained force from a microneedle (Alexa 555, yellow) as in Fig. 1 B. The whole spindle rotates and translates while the $k$-fiber proximal to the microneedle (white line, tracked) bends and lengthens compared with a control $k$-fiber (red line, tracked). Scale bar, $4 \mu \mathrm{m}$. See also Video 2. (C) Maps of the tracked k-fiber shapes and positions for control and manipulated k-fibers from B. Open circles indicate plus-end positions and filled circles indicate pole positions. The manipulated k-fiber (right) translates in the xy-plane and bends and lengthens over time; the control k-fiber (left) similarly translates, but does not lengthen. (D) Speed of proximal pole (left) and plus end (kinetochore, right) movement relative to the speed of microneedle movement within a half-spindle. Half-spindle movement is positively correlated with microneedle speed, indicating global dissipation of force (pole: Spearman $R=0.48, P=0.04$; plus end: Spearman $R=0.71, P=0.0007 ; n=18$ cells). (E) The $k$-fiber length as a function of time, normalized by subtracting the initial length at start of force application $(t=0)$ for $k$-fibers manipulated (right, black; $n=18$ cells), in the middle of the halfspindle (middle, blue; $n=13$ cells), and on the opposite side of the half-spindle (left, red; $n=18$ cells). The micromanipulated $k$-fiber lengthens persistently during force application while the other $k$-fibers grow and shrink, but do not systematically change length. (F) Average $k$-fiber lengths at start and end of force application as a function of $k$-fiber position in the half-spindle. The manipulated (Manip.) $k$-fiber (black, $n=18$ cells) significantly increased in length ( $P=$ 0.0002 , two-sided Wilcoxon signed-rank test), while the middle and outer $k$-fiber lengths remain unchanged $(P=0.73, n=13$ cells and $P=0.35, n=18$ cells, two-sided Wilcoxon signed-rank test). Plot shows mean \pm SEM. n.s., not significant. (G) Plot of average k-fiber growth rate for manipulated (Manip.) $k$-fibers (black, $n=18$ cells) compared with middle $k$-fibers (blue, $n=13$ cells) or outer $k$-fibers (red, $n=18$ cells) in the same half-spindle. Only the manipulated $k$-fiber lengthened significantly during force application while neighboring $k$-fibers continued oscillating between lengthening and shortening phases (manipulated k-fiber versus middle k-fiber "net," $\mathrm{P}=1.6 \times 10^{-5}$; manipulated k-fiber versus outer k-fiber net, $\mathrm{P}=1.4 \times 10-5 \times 10^{-5}$; middle $\mathrm{k}$-fiber net compared with outer $k$-fiber, $P=0.3$, two-sided Mann-Whitney $U$ test). The growth rate of the manipulated $k$-fiber was not significantly different from the growth rate of the middle $k$-fiber during just the growth phases of its oscillations (blue 'growth', $P=0.98$, two-sided Mann-Whitney $U$ test). Plot shows mean $\pm S E M$. $n$.s., not significant. $(\mathrm{H})$ Growth rate of the manipulated k-fiber as a function of the speed of microneedle movement. The growth rate of the manipulated k-fiber did not correlate with the speed of microneedle movement (Spearman $R=-.01, P=0.98, n=18$ cells). (I) Growth rate of the manipulated $k$-fiber as a function of distance between the microneedle center and the $\mathrm{k}$-fiber plus end. The growth rate of the manipulated $\mathrm{k}$-fiber does not correlate with the proximity of the microneedle to the plus end (Spearman $R=0.08, P=0.76, n=18$ cells). 
response to force from the microneedle, the spindle would either locally or globally deform (Fig. 2 A). In response to this perturbation, the spindle translated and rotated, with faster microneedle speeds giving rise to faster spindle speeds (Fig. 2, C and $\mathrm{D}$ ). Thus, we see global movement of the spindle in response to force. Yet, in these same spindles we also observed that $\mathrm{k}$-fibers lengthened, indicating that the spindle also locally responds to force (Fig. 2 E). During the pull, the manipulated k-fiber bent and lengthened by $4.1 \pm 0.8 \mu \mathrm{m}$; meanwhile, an unmanipulated $\mathrm{k}$-fiber in the same spindle half lengthened significantly less over the same duration (net k-fiber growth $0.03 \pm 0.32 \mu \mathrm{m}$, Mann-Whitney $U$ test, $\mathrm{P}=6 \times 10^{-5}$; Fig. $2 \mathrm{~F}$ ). Thus, force is dissipated locally by k-fiber bending and lengthening and globally by whole spindle movements.

The manipulated $k$-fiber grew at $1.6 \pm 0.3 \mu \mathrm{m} / \mathrm{min}$, a rate that was not significantly faster than that of its neighboring unmanipulated $\mathrm{k}$-fiber during the growth phases of its oscillations $(1.4 \pm 0.1 \mu \mathrm{m} / \mathrm{min}$, Mann-Whitney $U$ test, $\mathrm{P}=0.98$; Fig. $2 \mathrm{G}$ and Fig. S1 C). However, the manipulated k-fiber persistently lengthened (Fig. $2 \mathrm{E}$ and Fig. S1 D), with either undetectable or very transient shortening, for longer than typical metaphase oscillations (Wan et al., 2012; Civelekoglu-Scholey et al., 2013). There was no correlation between $\mathrm{k}$-fiber growth rate and pulling speed (Fig. $2 \mathrm{H}$ ), suggesting either that force was dissipated before reaching the $\mathrm{k}$-fiber's ends or that force does not regulate its maximum growth rate (Nicklas, 1983, 1988; Skibbens and Salmon, 1997; Betterton and McIntosh, 2013). Furthermore, the k-fiber growth rate did not vary with the proximity of the microneedle to the plus end (Spearman $\mathrm{R}$ coefficient $=0.08, \mathrm{P}=$ 0.76; Fig. 2 I), which we hypothesized would lead to more direct force transmission, consistent with force not regulating the k-fiber's maximum growth rate. Together, these findings indicate that individual $\mathrm{k}$-fibers remodel under sustained force for minutes by persistently lengthening. They also suggest that force inhibits their normal switching dynamics rather than substantially increasing their growth rate, which may serve as a protective mechanism to limit the rate of spindle deformations and thereby preserve spindle structure.

\section{Force on individual mammalian $\mathbf{k}$-fibers suppresses depolymerization at both ends without altering plus-end polymerization rates or inducing microtubule sliding} Metaphase mammalian k-fibers typically depolymerize at their minus-ends and switch between polymerizing and depolymerizing at their plus ends. Thus, force could lengthen k-fibers by increasing plus-end polymerization rates, by suppressing depolymerization at either end, by sliding microtubules within the k-fiber (Fig. $3 \mathrm{~A}$ ), or by a combination of these mechanisms. To determine the physical mechanism of $\mathrm{k}$-fiber lengthening under sustained force, we photomarked photoactivatable (PA)-GFPtubulin on a k-fiber before micromanipulation and tracked the photomark's position and size within the k-fiber (colabeled with silicon rhodamine [SiR]-tubulin; Fig. 3 B and Fig. S2) over time. In unmanipulated cells, photomarks fluxed toward the pole at a constant rate that reports on depolymerization at the minus end (Fig. 3 C; Mitchison, 1989). Upon external force from the microneedle, the photomark-to-pole distance remained constant
(Fig. 3 D), while the photomark-to-plus-end distance increased (Fig. 3 E). This response indicates that applied force suppresses microtubule depolymerization at $\mathrm{k}$-fiber minus ends and that $\mathrm{k}$-fibers lengthen by sustained polymerization at plus ends.

Mapping these findings to the previous experiment measuring $\mathrm{k}$-fiber lengthening (Fig. 2, E and G), we found that in the subset of $\mathrm{k}$-fibers that lengthened (15/18), the growth rate was $1.9 \pm 0.4 \mu \mathrm{m} / \mathrm{min}$, which is the rate of plus-end polymerization given that depolymerization at both ends is inhibited (Fig. 3, D and E). This is similar to the plus-end polymerization rate of neighboring unmanipulated $\mathrm{k}$-fibers during natural growth: lengthening at $1.4 \pm 0.1 \mu \mathrm{m} / \mathrm{min}$ (Fig. $2 \mathrm{G}$ ), while depolymerizing at the minus end at $\sim 0.5 \mu \mathrm{m} / \mathrm{min}$ results in a polymerization rate of $\sim 1.9 \mu \mathrm{m} / \mathrm{min}$ at plus ends (Mann-Whitney $U$ test, $\mathrm{P}=$ 0.55 ; Long et al., 2017). This indicates that the applied force does not increase mammalian k-fiber plus-end polymerization rates.

Notably, the average width of the photomark remained constant during manipulation (Fig. 3, F and G), indicating the microtubules do not detectably slide within the bundle. Thus, the k-fiber behaves as a single coordinated mechanical unit, rather than as microtubules that independently respond to force. Together, our findings indicate that individual $k$-fibers lengthen under force by remodeling their ends and not their bundle structure: force suppresses depolymerization locally at both plus and minus ends (Fig. 3), leads to persistent plus-end polymerization at a force-independent rate (Fig. 2 and Fig. 3), and does so with the $\mathrm{k}$-fiber responding as a single mechanical unit (Fig. 3). Thus, force is dissipated locally at k-fiber ends. This may limit force transmission to the rest of the spindle, thereby preserving overall $\mathrm{k}$-fiber and spindle architecture for proper chromosome segregation.

\section{Interfaces between mammalian $\mathbf{k}$-fibers and the kinetochore and pole are more robust than $\mathrm{k}$-fiber bundles under sustained force}

Finally, we asked how a k-fiber's structure and spindle connections changed over the $\sim 5-7-\mathrm{min}$ lifetime of its microtubules (Gorbsky and Borisy, 1989; Cassimeris et al., 1990; Zhai et al., 1995), since this could set a timescale for their response to force. We hypothesized that as microtubules turn over, the manipulated k-fiber could, for example, detach from the spindle or break (Fig. 4 A). Over all microneedle pulls (Fig. 2 and Fig. 3) that lasted several minutes, we never observed k-fiber detachment from the kinetochore or pole, indicating strong anchorage at those force-dissipating sites (Nicklas and Staehly, 1967; Begg and Ellis, 1979; Nicklas et al., 1982; Gatlin et al., 2010; Fong et al., 2017). Instead, k-fibers bent, lengthened (Fig. 2 and Fig. 3), and then in a subset of cells occasionally broke, $3.9 \pm 0.5 \mathrm{~min}$ after the start of pulling (Fig. 4 B and Fig. S3 A). To probe the mechanism of this breakage, we examined $\mathrm{k}$-fiber structure over time and the kinetics of breakage. The k-fibers that broke sustained high curvature for many minutes before breaking (Fig. $4 \mathrm{C}$ ) and reached a maximum curvature similar to those that did not (Fig. 4 D). Furthermore, k-fiber breakage kinetics appeared independent of the specific manner in which forces are exerted on the k-fiber: the time to breakage was similar when we moved the microneedle for a shorter time and held it in 
A

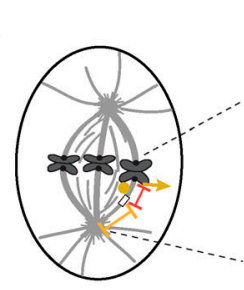

Response at

minus end

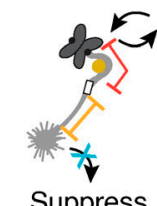

Suppress depolymerization

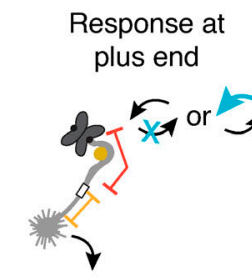

Suppress depolymerization or increase polymerization
Response

within k-fiber

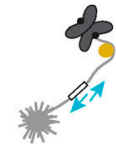

Microtubules slide
B
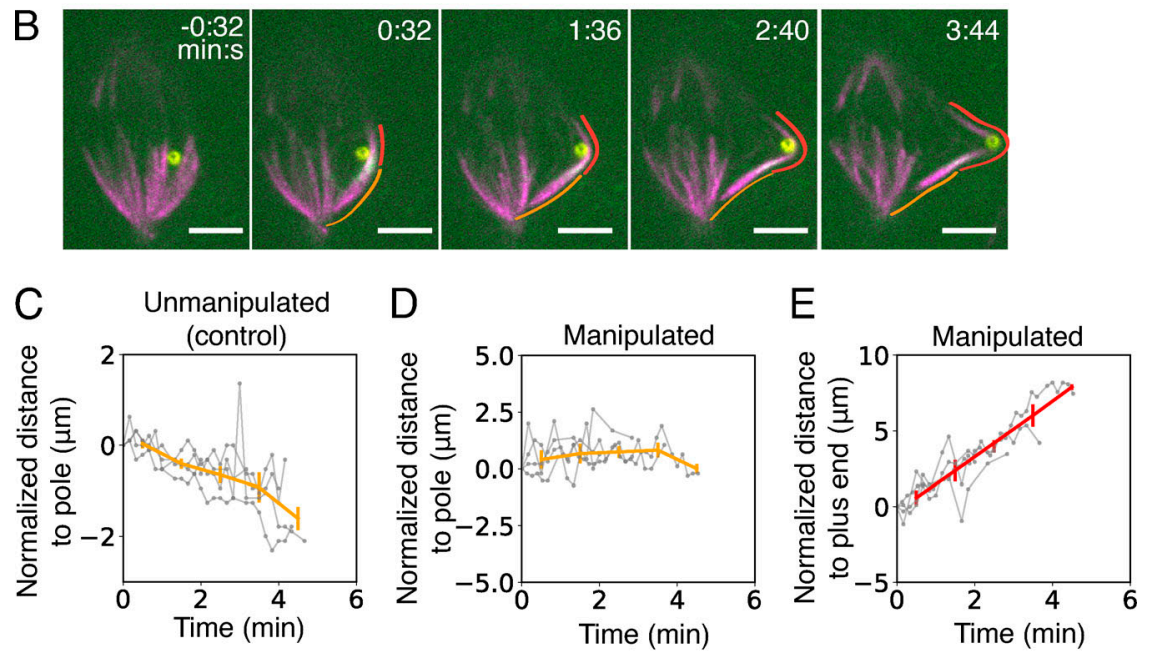

$\mathrm{F}$

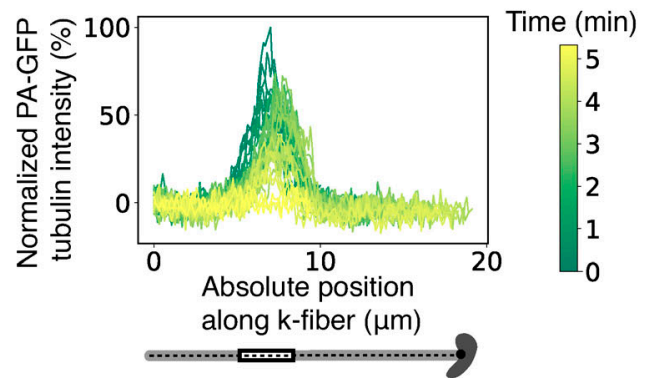

Figure 3. Force on individual mammalian k-fibers suppresses depolymerization at both ends without altering plus-end polymerization rates or inducing microtubule sliding. (A) Assay to determine the physical mechanism of $k$-fiber lengthening under force by tracking position of a photomark on the k-fiber during microneedle manipulation. Possible outcomes are shown, not mutually exclusive: the photomark could remain fixed relative to the pole, indicating a suppression of minus-end depolymerization (left, blue $\mathrm{X}$ ); the position of the photomark to the kinetochore could increase continuously, indicating a suppression of plus-end depolymerization or increase in plus-end polymerization rate (middle, blue $\mathrm{X}$ or arrow); or the photomark could remain in a fixed position but widen, indicating sliding of microtubules within the k-fiber (right, blue X). (B) Representative time-lapse images of photomark (PA-GFP-tubulin, white) during microneedle (Alexa 555, yellow) manipulation of a k-fiber (SiR-tubulin, magenta). The distance between the photomark and the pole remains constant (orange line), while the distance between the photomark and plus end increases (red line). Scale bar, $4 \mu \mathrm{m}$. See also Video 3. (C) Plot of the photomark to pole distance change over time due to flux of microtubules in unmanipulated cells, as a baseline ( $n=4$ cells). (D) Plot of the photomark to pole distance change during microneedle manipulation, showing that photomark movement poleward due to microtubule depolymerization is suppressed ( $n=4$ cells). (E) Plot of the photomark to plus end position distance change during microneedle manipulation, showing that $k$-fibers persistently polymerize at their plus ends under force ( $n=4$ cells). (F) Representative example of photomark intensity linescans over time during manipulation, from same cell as B. (G) Change in full width at half maximal photomark intensity at each time point during microneedle manipulation, showing that photomarks do not widen under force, and thus that there is no detectable microtubule sliding within the $\mathrm{k}$-fiber $(n=4$ cells). place, or pulled continuously for the entire duration of manipulation (Fig. 1 B and Fig. 4 E). Together, these findings suggest that the breakage process occurred gradually over sustained force, rather than rapidly by reaching an acute mechanical limit of k-fiber bending (Nicklas et al., 1989; Gittes et al., 1993; Ward et al., 2014; Schaedel et al., 2015). A k-fiber damage process that is gradual would promote breakage in response to sustained, but not transient, forces, setting a limiting timescale for restoring spindle structural homeostasis.

A possible model for gradual damage of the $\mathrm{k}$-fiber over minutes is loss of microtubules as they turn over and fail to replenish within the k-fiber. In addition to turnover, it is also possible that there are alterations to $k$-fiber microtubule structure that would lead to gradual damage. During these manipulations, we observe microtubule plus ends that appear to "splay" from the bundle near the needle in $80 \%$ of $\mathrm{k}$-fibers before breakage (Fig. 4, B and F), and when we can track plus ends after breakage, they fail to depolymerize (Fig. $4 \mathrm{G}$ ). This is in contrast to abruptly created $\mathrm{k}$-fiber plus ends that depolymerize within seconds (Spurck et al., 1990; Sikirzhytski et al., 2014; Elting et al., 2014) and suggests a change in local microtubule structure before breakage that stabilizes plus ends at the breakage site (Schaedel et al., 2015; Portran et al., 2017; Vemu et al., 2018; McNally and Roll-Mecak, 2018; Gasic and Mitchison, 2019). Together, these findings show how mammalian k-fibers gradually respond to and dissipate sustained forces over their microtubule's lifetime. They robustly remain attached at kinetochores, yet eventually they locally break in the middle of the bundle, thereby preserving connections of chromosomes to the spindle at the expense of nonessential direct connections to poles (Sikirzhytski et al., 2014; Elting et al., 2014).

\section{Local force dissipation by mammalian $k$-fibers maintains robust spindle structure}

In mammals, chromosome segregation is powered by dynamic k-fibers that both generate and respond to force. Here, we use microneedle manipulation to directly probe how k-fiber dynamics and structure respond to sustained force (Fig. 1). We 

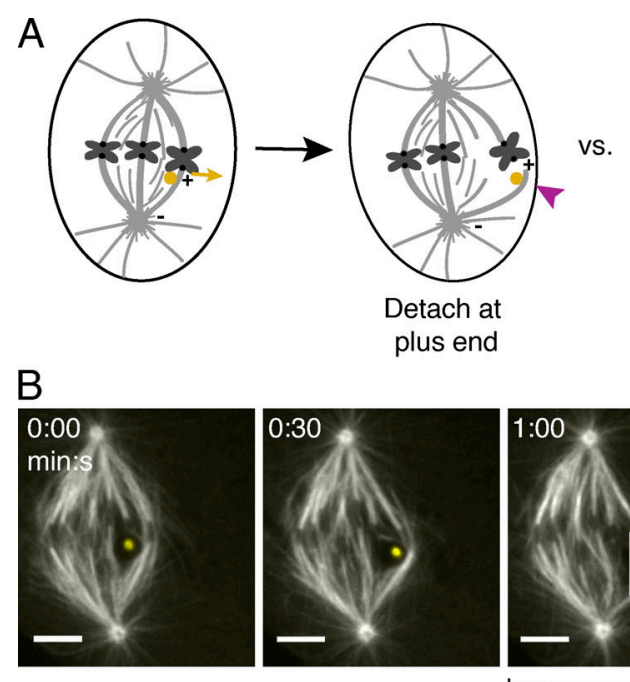

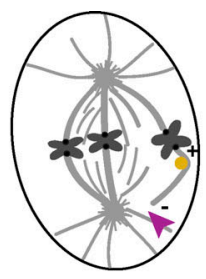

Detach at minus end

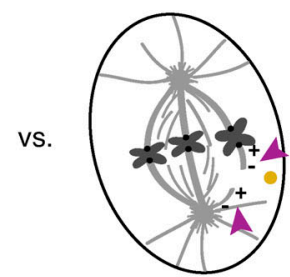

Break instead of detaching at ends
C

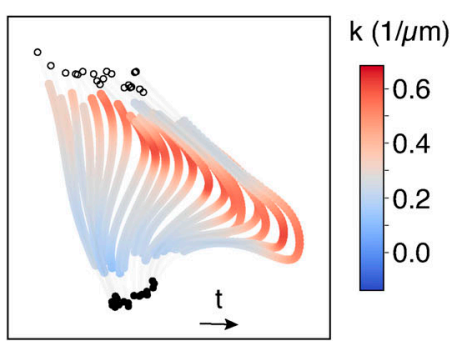

E

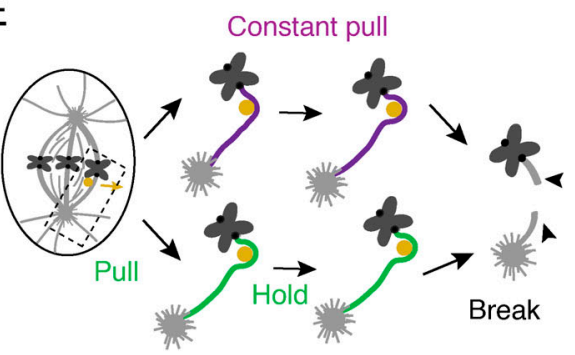

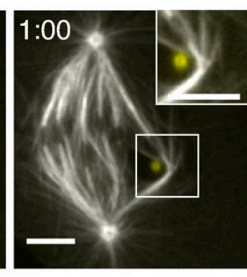

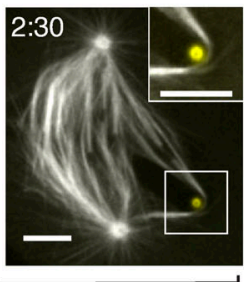

Splay

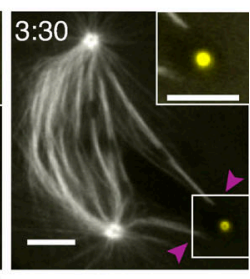

Break
G

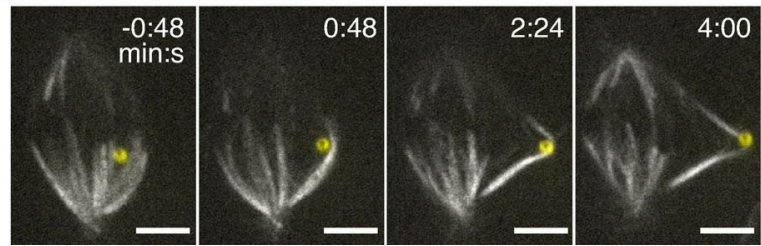

D

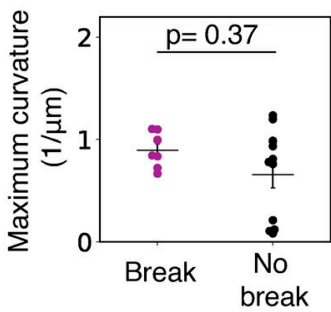

$\mathrm{F}$

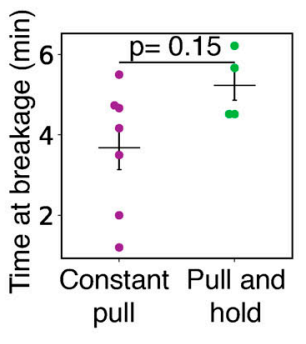

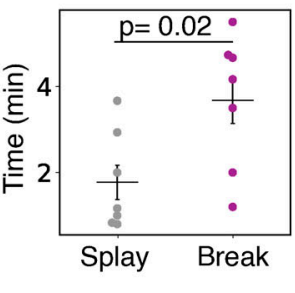

Figure 4. Interfaces between mammalian k-fibers and the kinetochore and pole are more robust than k-fiber bundles under sustained force. (A) Three example outcomes of force application (yellow arrow and circle) for several minutes are shown: the k-fiber could detach (purple arrowhead) from the kinetochore (second panel, "+"), the k-fiber could detach (purple arrowhead) from the pole (third panel, "-"), or the k-fiber could remain attached at its ends but break (purple arrowheads) in its center (fourth panel; vs., versus). (B) Representative time-lapse images of k-fiber (GFP-tubulin, white) bending, lengthening, and breaking under sustained force. Before the k-fiber breaks, microtubules appear (insets) on the outside of the deformed k-fiber near the area of high curvature next to the microneedle (Alexa 647, yellow). The break creates new microtubule bundle plus ends (purple arrowheads). Scale bar, $4 \mu \mathrm{m}$. See also Video 4. (c) Example map of local curvature ( $k$ ) along a $k$-fiber bundle during sustained microneedle manipulation. As the $k$-fiber bends over time, high curvature (dark red) increases near the microneedle and persists for many minutes before breakage occurs ( 3.5 min). Open circles indicate plus end positions, and filled circles indicate pole positions. (D) Maximum curvature along the k-fiber in the last tracked time point before breakage in cells with breakage events (purple, $n=6$ cells) or at the end of the manipulation for cells with no breakage (black; $n=11$ cells). Plot shows mean $\pm S E M(P=0.37$, two-sided Mann-Whitney $U$ test). (E) Cartoon of two different micromanipulation assays that lead to $k$-fiber breakage: microneedle is moved continuously at $5.2 \pm 0.2 \mu \mathrm{m} / \mathrm{min}$ for $3.1 \pm$ $0.3 \mathrm{~min}$ (top, purple) and microneedle is moved at $4.5 \pm 0.7 \mu \mathrm{m} / \mathrm{min}$ for $1.7 \pm 0.2 \mathrm{~min}$ and then held in place until breakage (bottom, green). Plot showing no significant difference in the time at breakage in each assay; plot shows mean \pm SEM ( $n=7$ cells and 4 cells, respectively; $P=0.15$, two-sided Mann-Whitney $U$ test). (F) Plot of the average time to a splaying event (where newly visible microtubules appear near the area of high curvature) and average time to breakage for the subset of cells in which both events occurred. Splaying events occurred significantly before breakage events (plot shows mean \pm SEM, $n=9$ cells, 
$P=0.02$, two-sided Wilcoxon signed-rank test). (G) Example time-lapse images of breakage event in which the newly created bundle plus ends (lower purple arrowhead) are highly stable and persist for minutes after breakage. This example cell is the same as the cell shown in Fig. 3 B, but here the cell is displaying the full response, including breakage. See also Video 5.

thereby define how the spindle's longest lived microtubule structure (Gorbsky and Borisy, 1989; Cassimeris et al., 1990; Zhai et al., 1995), the k-fiber, remodels under force, which is key for understanding spindle structural homeostasis. We find that individual $\mathrm{k}$-fibers respond to and dissipate sustained force by locally turning off microtubule depolymerization at both plus and minus ends (Figs. 2, 3, S1, and S2) and eventually breaking on the timescale of their microtubule turnover (Fig. 4 and Fig. S3). They do so without increasing their rate of plus-end polymerization (Fig. 2 and Fig. 3), without sliding their microtubules within the k-fiber (Fig. 2 and Fig. 3) and without detaching from kinetochores or poles (Fig. 4). Thus, how the $\mathrm{k}$-fiber responds-and does not respond-to force allows it to act as a single mechanical unit that can maintain its connections to chromosomes and preserve global spindle structure. The ability to directly exert force on the mammalian spindle is key to this work as it allowed us to clearly probe the feedback between force, structure, and dynamics in the spindle (Elting et al., 2018). Together, these findings suggest different physical mechanisms of local force dissipation as an engineering principle for the spindle to maintain its structure and function under sustained forces (Fig. 5). More broadly, this study provides a framework for understanding how the spindle remodels under force during chromosome segregation.

We show that mammalian k-fiber plus ends persistently polymerize at normal rates in response to applied force (Fig. 2 and Fig. 3). Microtubules attached to yeast kinetochore particles or subcomplexes in vitro polymerize under force at faster than normal rates, in addition to suppressing catastrophe and favoring rescue under force (Franck et al., 2007; Akiyoshi et al., 2010). In newt cells, force induces persistent $k$-fiber lengthening at normal k-fiber growth rates (Skibbens and Salmon, 1997), and our findings suggest that this may occur through regulation of dynamics at both ends. Overall, the force-velocity relationships at kinetochore-microtubule plus ends in vertebrate cells are remarkably similar to those of yeast kinetochores particles in vitro. The differences in whether the polymerization rate increases under force may stem from differences in applied forces, kinetochore or k-fiber architecture (Long et al., 2019), and additional regulation in cells or be related to the growth rates of microtubules in vivo already being close to their limits. The molecular basis of potential "governors" of $k$-fiber plus-end polymerization velocity has been a long-standing question (Nicklas, 1983; Betterton and McIntosh, 2013; Long et al., 2017), and our findings suggest that in mammals this molecular governor is not mechanically regulated. Notably, force not regulating mammalian k-fiber polymerization velocity (Fig. 2 and Fig. 3) could provide a protective upper limit to how fast the spindle can remodel. It also has implications for mechanical communication in the spindle, e.g., how force regulates kinetochoremicrotubule attachments (Li and Nicklas, 1995; Sarangapani and Asbury, 2014).

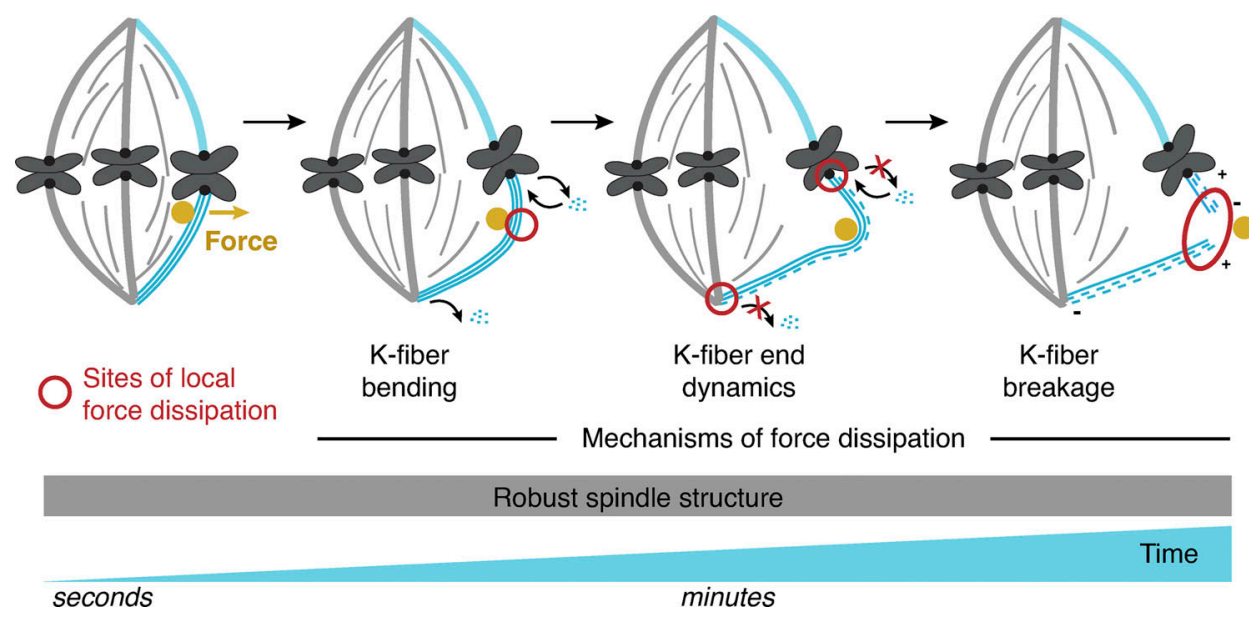

Figure 5. A model for local force dissipation by individual k-fibers to maintain robust mammalian spindle structure. Using micromanipulation to apply sustained forces (yellow circle, arrow) on individual mammalian k-fibers reveals that they locally dissipate force (red circles) using different physical mechanisms over different timescales (blue ramp, dashed lines indicate microtubule turnover) to robustly preserve global spindle structure (gray box). Key to this model is how k-fibers both remodel under and resist sustained force. The k-fibers remodel and locally dissipate force: they bend (second panel), lengthen through suppressing depolymerization at their plus and minus ends (third panel, small black "off" arrows with red X), and gradually break (fourth panel, new black plus and minus ends). In turn, $\mathrm{k}$-fibers also resist force to preserve spindle structure: they do not increase their polymerization rate (third panel, small black "on" arrow), slide their microtubules, or detach from kinetochores or poles under force. Note that for simplicity, we do not diagram whole spindle movements and only show individual microtubules for the manipulated k-fiber. Thus, local dissipation and isolation mechanisms together preserve mammalian spindle structure under sustained forces: the former limit how far and for how long forces can be transmitted across the spindle, while the latter limit the spindle's deformation rate and preserve k-fiber and spindle structure and their connections. Together, this model suggests local force dissipation at multiple sites as an engineering principle for the dynamic spindle and other cellular machines to robustly maintain their structure and function under force. 
We demonstrate that force not only regulates the dynamics of individual k-fibers' plus ends but also of their minus ends (Fig. 3). Thus, both k-fiber ends serve as sites of force dissipation, allowing forces exerted on k-fibers to be locally and robustly dissipated, thereby limiting disruption to the rest of the spindle. The fact that force regulates minus end dynamics of single k-fibers indicates that their regulation occurs at the level of the individual k-fiber and not globally as hypothesized when force was applied to the whole spindle (Dumont and Mitchison, 2009; Guild et al., 2017). Although we cannot exclude it, we did not detect force-induced polymerization at $\mathrm{k}$-fiber minus ends; thus, force dissipation also appears limited at minus ends. The microneedle approach we present here, combined with perturbations of microtubule regulators at minus ends (Ganem et al., 2005; Ganem and Compton, 2006), will be key in defining the molecular basis of the regulation of $k$-fiber minus end dynamics by force. Together, the response of individual $k$-fibers' dynamics to force, at both ends, allows each k-fiber to locally isolate and dissipate applied force while retaining its internal organization and global spindle structure. Thus, k-fiber end dynamics mechanically buffer global spindle deformations from local forces to maintain structural homeostasis (Maddox et al., 2003; Matos et al., 2009).

On longer timescales, we find that the k-fiber breaks under force, without detaching from the kinetochore or pole (Fig. 4 and Fig. S3). This is surprising as force-induced detachments from kinetochores occur in vitro (Akiyoshi et al., 2010) and in meiotic insect cells (Nicklas, 1967; Nicklas and Koch, 1969; Paliulis and Nicklas, 2004; Lin et al., 2018). This difference could, for example, arise from variations in force application, or in the physical properties or architectures of their kinetochores (Cheerambathur et al., 2017; Auckland et al., 2017; Agarwal et al., 2018; Yoo et al., 2018). Instead of detaching, the $\mathrm{k}$-fiber breaks on a timescale similar to that of its microtubule lifetime, suggesting that the $\mathrm{k}$-fiber's lifetime may limit the long-term impact force can have over spindle structure. Our findings suggest a model of gradual $\mathrm{k}$-fiber damage and that sustained force may not only regulate biochemistry at the $\mathrm{k}$-fiber's ends but also in its middle along the microtubule lattice (Fig. 4, F and G). Local defects in the lattice can replenish GTP-tubulin, creating stable sites for microtubule repair or enzymatic activities that may alter the physical properties of microtubules (Schaedel et al., 2015; Portran et al., 2017; Vemu et al., 2018; McNally and Roll-Mecak, 2018; Gasic and Mitchison, 2019). Looking forward, combining microneedle manipulation with different imaging methodologies that allow us to better capture the full spindle volume, such as lattice light-sheet imaging, will give us clearer insights into how $\mathrm{k}$-fiber structure changes under force. Under sustained force, $\mathrm{k}$-fiber attachments to chromosomes are prioritized over direct connections between chromosomes and poles, which are not necessary for segregation (Elting et al., 2014; Sikirzhytski et al., 2014) and thus may not be key for function.

Altogether, we show that mammalian spindles locally dissipate sustained force by remodeling k-fiber dynamics and structure through different physical mechanisms over time (Fig. 5). In principle, this can allow the spindle to preserve robust connections to chromosomes and maintain its structure under force throughout mitosis. Local dissipation of force limits its impact on the rest of the spindle, providing local isolation. In turn, the timescale of such dissipation limits the timescale of mechanical memory in the spindle. By regulating force dissipation, the spindle could set the impact force has on its structure over time to allow it to respond to different mechanical cues and perform different mechanical functions. Looking forward, it will be of interest to map how spindles with different $k$-fiber dynamics and structures across species dissipate and transmit force and thereby preserve their structural homeostasis (Nicklas and Staehly, 1967; Shimamoto and Kapoor, 2012; Itabashi et al., 2009; Crowder et al., 2015; Takagi et al., 2019). Finally, we note that the local force dissipation at multiple sites we observe in the spindle is a simple engineering principle by which a cellular structure may be mechanically robust, analogous to how structural engineers design sites of local force dissipation to make buildings and bridges robust to external forces.

\section{Materials and methods \\ Cell culture}

PtK2 cells were cultured in MEM (Invitrogen) supplemented with sodium pyruvate (Invitrogen), nonessential amino acids (Invitrogen), penicillin/streptomycin, and $10 \%$ qualified and heat-inactivated fetal bovine serum (Invitrogen) and maintained at $37^{\circ} \mathrm{C}$ and $5 \% \mathrm{CO}_{2}$. PtK2 cells stably expressing human GFP- $\alpha-$ tubulin (gift from Alexey Khodjakov, Wadsworth Center, Albany, NY) and PtK2 cells incubated with SiR-tubulin dye (Cytoskeleton, Inc.) were both used. PtK2 cells stably expressing human EYFP-Cdc20 (gift from Jagesh Shah, Harvard Medical School, Boston, MA) were used for Fig. 1 validation of microneedle manipulation. SiR-tubulin at $100 \mathrm{nM}$ and $10 \mu \mathrm{M}$ verapamil (Cytoskeleton, Inc.) were incubated with cells for $45 \mathrm{~min}$ before imaging for cells not expressing GFP-tubulin. For validation that the microneedle did not enter cells, PtK2 cells expressing GFP- $\alpha$-tubulin were treated with CellMask Orange dye diluted 1:1,000 (Thermo Fisher Scientific) for 5 min before immediate imaging with no wash step (Fig. 1 C). PtK1 cells stably expressing PA-GFP- $\alpha$-tubulin (gift from Alexey Khodjakov) were cultured in Ham's F12 media (Invitrogen) supplemented with penicillin/streptomycin and $10 \%$ qualified and heatinactivated fetal bovine serum and maintained at $37^{\circ} \mathrm{C}$ and $5 \%$ $\mathrm{CO}_{2}$. For photoactivation experiments, PtK1 PA-GFP-tubulin cells were colabeled with SiR-tubulin as described above to mark overall spindle structure. Control cells labeled with SiR-tubulin that did not undergo microneedle manipulation still exhibited chromosome oscillations and poleward microtubule flux at a rate of $0.40 \pm 0.06 \mu \mathrm{m} / \mathrm{min}$ (Fig. $3 \mathrm{C}$ ), indicating that this concentration and length of dye incubation did not suppress k-fiber microtubule dynamics in these cells.

\section{Microscopy}

Live cells were imaged using an inverted microscope (Eclipse Ti-E; Nikon) with a spinning disk confocal (CSU-X1; Yokogawa), head dichroic Semrock Di01-T405/488/568/647 for multicolor imaging, equipped with $405 \mathrm{~nm}$ (100 mW), $488 \mathrm{~nm}(120 \mathrm{~mW})$, 
$561 \mathrm{~nm}(150 \mathrm{~mW})$, and $642 \mathrm{~nm}(100 \mathrm{~mW})$ diode lasers, emission filters ET455/50M, ET525/50M, ET630/75M, and ET690/50M for multicolor imaging, and an iXon3 camera (Andor Technology) operated by MetaMorph 7.7.8.0 (Molecular Devices). Cells were imaged with a $100 \times 1.45 \mathrm{Ph} 3$ oil objective and $1.5 \times$ lens every $10 \mathrm{~s}$, acquiring three z-planes spaced 0.35-0.50 $\mu \mathrm{m}$ apart with a PZ-2000 z-piezo stage (ASI). Cells were imaged in a stage-top incubation chamber (Tokai Hit) with the top lid removed and maintained at $30^{\circ} \mathrm{C}$. Cells were plated on glassbottomed, 35-mm dishes coated with poly-D-lysine (MatTek Corporation) and imaged in $\mathrm{CO}_{2}$-independent MEM (Invitrogen) supplemented as for PtK2 cell culture as described above. Photoactivation was performed using a MicroPoint pulsed laser system (Andor) to deliver several $3 \mathrm{~ns}, 20 \mathrm{~Hz}$ pulses of $405 \mathrm{~nm}$ light to activate PA-GFP-tubulin (Fig. 3).

\section{Microneedle manipulation}

Microneedle manipulation was adapted for use in mammalian spindles (Suresh et al., 2020) for sustained periods of many minutes by optimizing needle dimensions, contact geometry, and speed of motion to minimize cellular damage.

\section{Preparation of microneedles}

Glass capillaries with an inner and outer diameter of 1 and 0.58 $\mathrm{mm}$, respectively (1B100-4 and 1B100F-4; World Precision Instruments), were used to create microneedles using a pipette puller (P-87; Sutter Instruments). For a ramp value of 504 (specific to the type of glass capillary and micropipette puller), we used the following settings: heat $=509$, pull $=70$, velocity $=$ 45 , delay $=90$, pressure $=200$, prescribed to generate microneedles of $0.2 \mu \mathrm{m}$ outer tip diameter (Sutter Instruments Pipette Cookbook). The measured diameter of the microneedle in the $\mathrm{z}$-plane of the manipulated k-fiber was $1.2 \pm 0.1 \mu \mathrm{m}$ (the tip was placed deeper than the k-fiber to ensure that it would not slip during movement). Microneedles with longer tapers and smaller tips than noted above were more likely to rupture the cell membrane. Microneedles were bent $\sim 1.5 \mathrm{~mm}$ away from their tip at a $45^{\circ}$ angle by using a microforge (Narishige International). This allowed for microneedles placed in the manipulator at a $45^{\circ}$ angle to approach the cell vertically and minimize the overall surface area of contact between the microneedle and the cell membrane.

Microneedles used for manipulation were coated with BSA Alexa Fluor 647 (A-34785; Invitrogen) or 555 conjugate (A34786; Invitrogen) by soaking in the solution for $60 \mathrm{~s}$ before imaging (Sasaki et al., 2012): BSA-Alexa 647 and sodium azide (Nacalai Tesque) were dissolved in $0.1 \mathrm{M}$ phosphate-buffered saline at the final concentration of $0.02 \%$ and $3 \mathrm{mM}$, respectively. Tip labeling was critical toward improving cell heath during sustained manipulations because it allowed us to better visualize the microneedle tip in fluorescence along with the spindle and prevented us from going too deeply into the cell, thereby causing rupture.

\section{Selection of cells}

Cells for micromanipulation were chosen that were at metaphase, flat, and had a spindle with both poles in the same focal plane. These criteria were important for pulling on single $\mathrm{k}$-fibers close to the top of the cell and simultaneously being able to image the whole spindle's response over several minutes of manipulation. Cells were included in our datasets if they did not appear negatively affected by micromanipulation. We did not include cells that underwent sudden and continuous blebbing upon microneedle contact, cells with spindles that started to collapse during manipulation, cells with decondensed chromosomes, or cells that entered anaphase during manipulation. We were often not able to follow cells after needle removal to assess rates of anaphase entry since in long manipulation experiments the cell and needle had significant time to interact and removing the needle often caused focal plane changes or detachment of the cell from the dish. In shorter duration manipulation experiments (12-60 s), $20 \%$ of cells underwent anaphase after needle removal. Short manipulations were likely less disruptive since the microneedle had shorter time to adhere to the cell (Suresh et al., 2020).

\section{Manipulation}

Manipulations were performed in 3D using an xyz stepper micromanipulator (MP-225; Sutter Instruments). A three-knob controller (ROE-200; Sutter Instruments) connected to the manipulator and controller (MPC-200; Sutter Instruments) allowed fine manual movements and was used to find and position the microneedle before imaging. To find and position the microneedle, we first located and centered the microneedle tip in the field of view using a low-magnification objective $(20 \times 0.5 \mathrm{NA}$ $\mathrm{Phl}$ air). We placed the microneedle in focus just above the coverslip before switching to a $100 \times 1.45 \mathrm{NA} \mathrm{Ph} 3$ oil objective and refined the xyz position of the microneedle to be right above a cell of interest, using the Phl phase ring to confirm microneedle position (phase ring mismatch visually highlights the position of the glass microneedle).

Upon choosing a cell to manipulate, we identified an outer $\mathrm{k}$-fiber in a plane close to the top of the cell and focused on this k-fiber. Next, we slowly brought the microneedle down into the cell using the fluorescent label of the microneedle tip to inform on its position until just deeper than the k-fiber of interest. If the microneedle's position was too far away from the k-fiber of interest, we slowly moved the microneedle out of the cell, adjusted its xy position, and brought it back down into the cell. Through this iterative process, we could correctly position the microneedle such that it was inside the spindle, next to the outer $\mathrm{k}$-fiber.

Once the microneedle was positioned next to an outer $\mathrm{k}$-fiber near the top of the cell, it was moved in a direction that is roughly perpendicular $\left(\sim 60-90^{\circ}\right)$ from the spindle's long axis using Multi-Link software (Sutter Instruments). We wrote a custom program to take as inputs the desired angle, duration, and distance for the microneedle movement and then output a set of instructions in steps, xy positions, and delays for the Multi-Link software to achieve to desired movement. For all manipulations except those in Fig. 4 E, we moved the microneedle at $5.2 \pm 0.2 \mu \mathrm{m} / \mathrm{min}$ for $3.1 \pm 0.3 \mathrm{~min}$ (Fig. $1 \mathrm{~B}$ ). For the "pull-and-hold" experiments, we moved the microneedle at $4.5 \pm$ $0.7 \mu \mathrm{m} / \mathrm{min}$ for $1.7 \pm 0.2 \mathrm{~min}$ and then held in place until 
breakage (Fig. 4 E). At the end of the manipulation, the microneedle was manually removed from the cell in the $\mathrm{z}$ axis slowly $(<5 \mu \mathrm{m} / \mathrm{min})$ to try to avoid membrane rupture or cell detachment from the coverslip.

\section{Tracking of spindle features}

For all analyses (Figs. 2, 3, and 4), k-fibers were manually tracked in Fiji version 2.0.0-rc-68/1.52g (Schindelin et al., 2012) by drawing segmented lines along maximum intensity projections of three z-planes of the fluorescent image with "spline fitting" checked. Splines were drawn from the edge of the tubulin signal at the plus-end to the center of the area of high tubulin intensity at the pole since we cannot determine specifically the location of the minus-end of the $\mathrm{k}$-fiber. Spline $\mathrm{x}$ and $\mathrm{y}$ coordinates were saved in CSV files using a custom macro in Fiji and imported into Python. All subsequent analysis and plotting were performed in Python. Microneedle position was tracked using the mTrackJ plugin (Meijering et al., 2012) in Fiji using the "snap to bright centroid" feature, and coordinates were saved in CSV files and imported into Python for further analysis.

\section{Quantification of spindle features}

Pole and kinetochore position were calculated using the $\mathrm{x}$ and $\mathrm{y}$ coordinates of the point at the end of the spline that terminated at the pole and kinetochore, respectively. Time $t=0$ was set to the first frame after the start of microneedle movement. Pole, microneedle, and kinetochore speeds were calculated using the average displacement of the ends of the spline or center of the microneedle position over time (Fig. 2, D and H). The k-fiber length and net growth rate were calculated using the length of the spline over time and with linear regression from the start and end of the manipulation (Fig. 2, E-I). For the analysis of $\mathrm{k}$-fiber growth rate of unmanipulated $\mathrm{k}$-fibers specifically during the growth phase (Fig. $2 \mathrm{G}$ ) and for the quantification of the duration of $\mathrm{k}$-fiber lengthening phases (Fig. S1 D), the start and end points were selected manually. The $k$-fiber lengthening was scored as a period of at least three time points in which the $\mathrm{k}$-fiber lengthened without interruption by more than one time point in which the length decreased. The distance between the microneedle and plus end was calculated as the linear distance between the center of the microneedle centroid and the plus end terminus of the spline (Fig. 2 I). Microtubule splaying was manually scored as the first frame in which new microtubule density appeared on the side of the k-fiber near the point of high curvature (Fig. 4, B and F). These events occurred within one time point $(<10 \mathrm{~s})$; thus, their dynamics of appearance could not be carefully characterized under these imaging conditions. The $\mathrm{k}$-fiber breakage was manually scored as the first frame in which the two k-fiber pieces moved in an uncorrelated manner (Fig. 4, B and E-G).

\section{Photomark analysis}

For photomark analysis, splines were tracked on maximum intensity projections of three z-planes using the 647 channel ( $\mathrm{SiR}$ tubulin label) and then that spline with a thickness of 5 pixels was used to calculate the intensity in the 488 channel (PA-GFPtubulin) at each point using a custom-written macro in Fiji, with all subsequent analysis in Python. Photomark position over time was calculated using the position along the curved $\mathrm{k}$-fiber spline at which the maximum intensity value occurred after masking bright intensity directly at the pole that was separate from the photomark signal (Fig. 3, C-E). Points were only included if the photomark remained in focus above background fluorescence. The k-fiber intensity was normalized to the average intensity of the k-fiber in the time point before photomarking to identify the peak; however, no intensity measurements were performed due to fluctuation of the $\mathrm{k}$-fiber in the $\mathrm{z}$ axis beyond the three $\mathrm{z}$-planes measured. For calculation of photomark width (Fig. 3 F), Gaussian fitting was performed on the normalized k-fiber intensities, and the full width at the half maximum intensity (FWHM) was calculated using the width of the distribution $(\sigma)$ obtained from the fit, as per FWHM $=2 \sqrt{2 \ln 2 \sigma}$ (Fig. $3 \mathrm{G}$ ) for the subset of time points where the Gaussian function could fit the data.

\section{Curvature analysis}

For curvature analysis (Fig. 4, C and D), local radius of curvature (in micrometers) was calculated by inscribing a circle through three points spaced by an interval of $1.5 \mu \mathrm{m}$ along the spline using a custom Python script. This radius was used to calculate curvature $(1 / \mu \mathrm{m})$ by taking the inverse.

\section{Video preparation}

Videos were formatted for publication using Fiji and set to play at similar rates relative to real time using a frame rate of 30 frames/s (Video 1) or 15 frames/s (Videos 2, 3, 4, and 5).

\section{Statistical analysis}

Data are reported as mean \pm SEM where indicated. All statistical testing was performed using the Python SciPy statistical package in Python. Two-sided Mann-Whitney $U$ testing was used to compare independent samples, whereas Wilcoxon signed-rank tests were used to compare paired datasets since we did not test whether assumptions for normality were met due to low sample size. Correlations were examined by calculating the Spearman rank-order correlation coefficient, and no outliers were removed. Because of the technical challenges of these experiments, sample sizes are small. We used $\mathrm{P}<0.05$ as the threshold for statistical significance and have directly indicated in the figures and figure legends the $P$ value and $n$, where $n$ refers to the number of cells. We have not performed statistical analysis for experiments with $n \leq 4$ (Fig. 3). No statistical methods were used to predetermine sample size. The experiments were not randomized.

\section{Online supplemental material}

Fig. S1 shows two additional examples of $\mathrm{k}$-fiber lengthening during force from microneedle manipulation as well as quantification of the duration of $k$-fiber lengthening in control versus manipulated k-fibers in the same half-spindle. Fig. S2 shows an additional example of photomark movement on a manipulated $\mathrm{k}$-fiber as it lengthens, consistent with new addition of tubulin at the plus and suppression of depolymerization at plus- and minus-ends. Fig. S3 reports that the average duration of manipulation in cells in which $\mathrm{k}$-fiber breakage occurred was slightly longer than in those with no breakage and provides two additional example images of 
k-fiber breakage events. Video 1 shows an example of the microneedle manipulation assay to reversibly exert sustained force on individual mammalian $k$-fibers. Video 2 provides an example of $\mathrm{k}$-fiber lengthening in response to sustained force from the microneedle. Video 3 shows how a photomark moves on a manipulated $\mathrm{k}$-fiber as it lengthens, demonstrating growth from the plus end and suppression of depolymerization at both ends. Video 4 provides an example of a $\mathrm{k}$-fiber breaking under force from the microneedle. Video 5 shows another example of $k$-fiber breakage under force and that the new $\mathrm{k}$-fiber fragment that is not attached to the kinetochore can persist after breakage.

\section{Acknowledgments}

We thank Le Paliulis and Yuta Shimamoto for critical microneedle manipulation advice, and Alan Verkman's laboratory for the use of their microforge. We thank Alexey Khodjakov for the gift of PtK2 GFP- $\alpha$-tubulin and PtK1 PA-GFP- $\alpha$-tubulin cell lines and Jagesh Shah for the gift of the PtK2 EYFP-Cdc20 cell line. We thank Ted Salmon for conversations that inspired this work. We thank David Agard, Maya Anjur-Dietrich, Charles Asbury, Arshad Desai, Wallace Marshall, Tim Mitchison, Dave Morgan, Max Nachury, Dan Needleman, Adair Oesterle, Ron Vale, Orion Weiner, and members of the Fred Chang and S. Dumont laboratories for helpful discussions.

This work was supported by National Institutes of Health DP2GM119177 and R01GM134132, National Science Foundation (NSF) Faculty Early Career Development Program 1554139, NSF Center for Cellular Construction DBI-1548297, the Rita Allen Foundation and Searle Scholars Program (to S. Dumont), NSF Graduate Research Fellowships Program (to A.F. Long and P. Suresh), and a University of California, San Francisco MoritzHeyman Discovery Fellowship and University of California, San Francisco Lloyd Kozloff Fellowship (to A.F. Long). S. Dumont is a Chan Zuckerberg Biohub investigator.

The authors declare no competing financial interests.

Author contributions: Conceptualization, A.F. Long, P. Suresh, and S. Dumont; Methodology, A.F. Long, P. Suresh; Investigation, A.F. Long and P. Suresh; Data Curation, A.F. Long and P. Suresh; Software, A.F. Long and P. Suresh; Writing Original Draft, A.F. Long; Writing - Review and Editing, A.F. Long, P. Suresh, and S. Dumont; Visualization, A.F. Long; Funding Acquisition, S. Dumont.

Submitted: 20 November 2019

Revised: 16 March 2020

Accepted: 27 April 2020

\section{References}

Agarwal, S., K.P. Smith, Y. Zhou, A. Suzuki, R.J. McKenney, and D. Varma. 2018. Cdtl stabilizes kinetochore-microtubule attachments via an Aurora B kinase-dependent mechanism. J. Cell Biol. 217:3446-3463. https:// doi.org/10.1083/jcb.201705127

Akiyoshi, B., K.K. Sarangapani, A.F. Powers, C.R. Nelson, S.L. Reichow, H. Arellano-Santoyo, T. Gonen, J.A. Ranish, C.L. Asbury, and S. Biggins. 2010. Tension directly stabilizes reconstituted kinetochore-microtubule attachments. Nature. 468:576-579. https://doi.org/10.1038/nature09594
Alushin, G.M., G.C. Lander, E.H. Kellogg, R. Zhang, D. Baker, and E. Nogales 2014. High-resolution microtubule structures reveal the structural transitions in a $\beta$-tubulin upon GTP hydrolysis. Cell. 157:1117-1129. https://doi.org/10.1016/j.cell.2014.03.053

Auckland, P., N.I. Clarke, S.J. Royle, and A.D. McAinsh. 2017. Congressing kinetochores progressively load Ska complexes to prevent forcedependent detachment. J. Cell Biol. 216:1623-1639. https://doi.org/10 $.1083 /$ jcb.201607096

Bakhoum, S.F., and D.A. Compton. 2012. Kinetochores and disease: keeping microtubule dynamics in check!. Curr. Opin. Cell Biol. 24:64-70. https:// doi.org/10.1016/j.ceb.2011.11.012

Begg, D.A., and G.W. Ellis. 1979. Micromanipulation studies of chromosome movement. I. Chromosome-spindle attachment and the mechanical properties of chromosomal spindle fibers. J. Cell Biol. 82:528-541. https://doi.org/10.1083/jcb.82.2.528

Betterton, M.D., and J.R. McIntosh. 2013. Regulation of chromosome speeds in mitosis. Cell. Mol. Bioeng. 6:418-430. https://doi.org/10.1007/s12195-013 $-0297-4$

Cassimeris, L., and E.D. Salmon. 1991. Kinetochore microtubules shorten by loss of subunits at the kinetochores of prometaphase chromosomes. J. Cell Sci. 98:151-158.

Cassimeris, L., C.L. Rieder, G. Rupp, and E.D. Salmon. 1990. Stability of microtubule attachment to metaphase kinetochores in PtK1 cells. J. Cell Sci. 96:9-15.

Cheerambathur, D.K., B. Prevo, N. Hattersley, L. Lewellyn, K.D. Corbett, K. Oegema, and A. Desai. 2017. Dephosphorylation of the Ndc80 Tail Stabilizes Kinetochore-Microtubule Attachments via the Ska Complex. Dev. Cell. 41:424-437.e4. https://doi.org/10.1016/j.devcel.2017.04.013

Cheeseman, I.M., and A. Desai. 2008. Molecular architecture of the kinetochoremicrotubule interface. Nat. Rev. Mol. Cell Biol. 9:33-46. https://doi.org/10 $.1038 / \mathrm{nrm} 2310$

Civelekoglu-Scholey, G., B. He, M. Shen, X. Wan, E. Roscioli, B. Bowden, and D. Cimini. 2013. Dynamic bonds and polar ejection force distribution explain kinetochore oscillations in PtK1 cells. J. Cell Biol. 201:577-593. https://doi.org/10.1083/jcb.201301022

Crowder, M.E., M. Strzelecka, J.D. Wilbur, M.C. Good, G. von Dassow, and R. Heald. 2015. A comparative analysis of spindle morphometrics across metazoans. Curr. Biol. 25:1542-1550. https://doi.org/10.1016/j.cub.2015.04.036

De Brabander, M., G. Geuens, R. Nuydens, R. Willebrords, F. Aerts, J. De Mey, and J.R. McIntosh. 1986. Microtubule dynamics during the cell cycle: the effects of taxol and nocodazole on the microtubule system of Pt K2 cells at different stages of the mitotic cycle. Int. Rev. Cytol. 101:215-274. https://doi.org/10.1016/S0074-7696(08)60250-8

Dogterom, M., J.W.J. Kerssemakers, G. Romet-Lemonne, and M.E. Janson. 2005. Force generation by dynamic microtubules. Curr. Opin. Cell Biol. 17:67-74. https://doi.org/10.1016/j.ceb.2004.12.011

Dumont, S., and T.J. Mitchison. 2009. Compression regulates mitotic spindle length by a mechanochemical switch at the poles. Curr. Biol. 19: 1086-1095. https://doi.org/10.1016/j.cub.2009.05.056

Dumont, S., E.D. Salmon, and T.J. Mitchison. 2012. Deformations within moving kinetochores reveal different sites of active and passive force generation. Science. 337:355-358. https://doi.org/10.1126/science.1221886

Elting, M.W., C.L. Hueschen, D.B. Udy, and S. Dumont. 2014. Force on spindle microtubule minus ends moves chromosomes. J. Cell Biol. 206:245-256. https://doi.org/10.1083/jcb.201401091

Elting, M.W., P. Suresh, and S. Dumont. 2018. The Spindle: Integrating Architecture and Mechanics across Scales. Trends Cell Biol. 28:896-910. https://doi.org/10.1016/j.tcb.2018.07.003

Fong, K.K., K.K. Sarangapani, E.C. Yusko, M. Riffle, A. Llauró, B. Graczyk, T.N. Davis, and C.L. Asbury. 2017. Direct measurement of microtubule attachment strength to yeast centrosomes. Mol. Biol. Cell. 28:1853-1861. https://doi.org/10.1091/mbc.e17-01-0034

Franck, A.D., A.F. Powers, D.R. Gestaut, T. Gonen, T.N. Davis, and C.L. Asbury. 2007. Tension applied through the Daml complex promotes microtubule elongation providing a direct mechanism for length control in mitosis. Nat. Cell Biol. 9:832-837. https://doi.org/10.1038/ncb1609

Ganem, N.J., and D.A. Compton. 2006. Functional roles of poleward microtubule flux during mitosis. Cell Cycle. 5:481-485. https://doi.org/10.4161/ c. 5.5.2519

Ganem, N.J., K. Upton, and D.A. Compton. 2005. Efficient mitosis in human cells lacking poleward microtubule flux. Curr. Biol. 15:1827-1832. https:// doi.org/10.1016/j.cub.2005.08.065

Gasic, I., and T.J. Mitchison. 2019. Autoregulation and repair in microtubule homeostasis. Curr. Opin. Cell Biol. 56:80-87. https://doi.org/10.1016/j .ceb.2018.10.003 
Gatlin, J.C., A. Matov, G. Danuser, T.J. Mitchison, and E.D. Salmon. 2010. Directly probing the mechanical properties of the spindle and its matrix. J. Cell Biol. 188:481-489. https://doi.org/10.1083/jcb.200907110

Gittes, F., B. Mickey, J. Nettleton, and J. Howard. 1993. Flexural rigidity of microtubules and actin filaments measured from thermal fluctuations in shape. J. Cell Biol. 120:923-934. https://doi.org/10.1083/jcb.120.4.923

Gorbsky, G.J., and G.G. Borisy. 1989. Microtubules of the kinetochore fiber turn over in metaphase but not in anaphase. J. Cell Biol. 109:653-662. https://doi.org/10.1083/jcb.109.2.653

Grishchuk, E.L. 2017. Biophysics of Microtubule End Coupling at the Kinetochore. In Centromeres and Kinetochores. B.E. Black, editor. Springer Nature Switzerland AG, Basel. 397-428. https://doi.org/10.1007/978-3 $-319-58592-5+17$

Grishchuk, E.L., M.I. Molodtsov, F.I. Ataullakhanov, and J.R. McIntosh. 2005. Force production by disassembling microtubules. Nature. 438:384-388. https://doi.org/10.1038/nature04132

Guild, J., M.B. Ginzberg, C.L. Hueschen, T.J. Mitchison, and S. Dumont. 2017. Increased lateral microtubule contact at the cell cortex is sufficient to drive mammalian spindle elongation. Mol. Biol. Cell. 28:1975-1983. https://doi.org/10.1091/mbc.e17-03-0171

Inoué, S., and E.D. Salmon. 1995. Force generation by microtubule assembly/ disassembly in mitosis and related movements. Mol. Biol. Cell. 6: 1619-1640. https://doi.org/10.1091/mbc.6.12.1619

Itabashi, T., J. Takagi, Y. Shimamoto, H. Onoe, K. Kuwana, I. Shimoyama, J. Gaetz, T.M. Kapoor, and S. Ishiwata. 2009. Probing the mechanical architecture of the vertebrate meiotic spindle. Nat. Methods. 6:167-172. https://doi.org/10.1038/nmeth.1297

Jaqaman, K., E.M. King, A.C. Amaro, J.R. Winter, J.F. Dorn, H.L. Elliott, N. McHedlishvili, S.E. McClelland, I.M. Porter, M. Posch, et al. 2010. Kinetochore alignment within the metaphase plate is regulated by centromere stiffness and microtubule depolymerases. J. Cell Biol. 188 665-679. https://doi.org/10.1083/jcb.200909005

Khodjakov, A., and C.L. Rieder. 1996. Kinetochores moving away from their associated pole do not exert a significant pushing force on the chromosome. J. Cell Biol. 135:315-327. https://doi.org/10.1083/jcb.135.2.315

Koshland, D.E., T.J. Mitchison, and M.W. Kirschner. 1988. Polewards chromosome movement driven by microtubule depolymerization in vitro. Nature. 331:499-504. https://doi.org/10.1038/331499a0

Li, X., and R.B. Nicklas. 1995. Mitotic forces control a cell-cycle checkpoint. Nature. 373:630-632. https://doi.org/10.1038/373630a0

Lin, N.K.H., R. Nance, J. Szybist, A. Cheville, and L.V. Paliulis. 2018. Micromanipulation of Chromosomes in Insect Spermatocytes. J. Vis. Exp. 57359. https://doi.org/10.3791/57359

Long, A.F., D.B. Udy, and S. Dumont. 2017. Hecl Tail Phosphorylation Differentially Regulates Mammalian Kinetochore Coupling to Polymerizing and Depolymerizing Microtubules. Curr. Biol. 27:1692-1699.e3. https://doi.org/10.1016/j.cub.2017.04.058

Long, A.F., J. Kuhn, and S. Dumont. 2019. The mammalian kinetochoremicrotubule interface: robust mechanics and computation with many microtubules. Curr. Opin. Cell Biol. 60:60-67. https://doi.org/10.1016/ .ceb.2019.04.004

Maddox, P., A. Straight, P. Coughlin, T.J. Mitchison, and E.D. Salmon. 2003 Direct observation of microtubule dynamics at kinetochores in Xenopus extract spindles: implications for spindle mechanics. J. Cell Biol. 162: 377-382. https://doi.org/10.1083/jcb.200301088

Matos, I., A.J. Pereira, M. Lince-Faria, L.A. Cameron, E.D. Salmon, and H. Maiato. 2009. Synchronizing chromosome segregation by fluxdependent force equalization at kinetochores. J. Cell Biol. 186:11-26. https://doi.org/10.1083/jcb.200904153

McDonald, K.L., E.T. O'Toole, D.N. Mastronarde, and J.R. McIntosh. 1992. Kinetochore microtubules in PTK cells. J. Cell Biol. 118:369-383. https:// doi.org/10.1083/jcb.118.2.369

McEwen, B.F., A.B. Heagle, G.O. Cassels, K.F. Buttle, and C.L. Rieder. 1997. Kinetochore fiber maturation in PtKl cells and its implications for the mechanisms of chromosome congression and anaphase onset. J. Cell Biol. 137:1567-1580. https://doi.org/10.1083/jcb.137.7.1567

McNally, F.J., and A. Roll-Mecak. 2018. Microtubule-severing enzymes: From cellular functions to molecular mechanism. J. Cell Biol. 217:4057-4069. https://doi.org/10.1083/jcb.201612104

Meijering, E., O. Dzyubachyk, and I. Smal. 2012. Methods for cell and particle tracking. In Methods in Enzymology. Volume 505. P. M. Conn, editor. Academic Press Inc., San Diego. 183-200.

Mitchison, T.J.. 1989. Polewards microtubule flux in the mitotic spindle: evidence from photoactivation of fluorescence. J. Cell Biol. 109:637-652. https://doi.org/10.1083/jcb.109.2.637
Mitchison, T., L. Evans, E. Schulze, and M. Kirschner. 1986. Sites of microtubule assembly and disassembly in the mitotic spindle. Cell. 45: 515-527. https://doi.org/10.1016/0092-8674(86)90283-7

Monda, J.K., and I.M. Cheeseman. 2018. The kinetochore-microtubule interface at a glance. J. Cell Sci. 131. jcs214577. https://doi.org/10.1242/jcs .214577

Nicklas, R.B.. 1967. Chromosome micromanipulation. II. Induced reorientation and the experimental control of segregation in meiosis. Chromosoma. 21:17-50. https://doi.org/10.1007/BF00330545

Nicklas, R.B.. 1983. Measurements of the force produced by the mitotic spindle in anaphase. J. Cell Biol. 97:542-548. https://doi.org/10.1083/jcb .97 .2 .542

Nicklas, R.B.. 1988. The forces that move chromosomes in mitosis. Annu. Rev. Biophys. Biophys. Chem. 17:431-449. https://doi.org/10.1146/annurev.bb .17 .060188 .002243

Nicklas, R.B.. 1997. How cells get the right chromosomes. Science. 275:632-637. https://doi.org/10.1126/science.275.5300.632

Nicklas, R.B., and C.A. Koch. 1969. Chromosome micromanipulation. 3. Spindle fiber tension and the reorientation of mal-oriented chromosomes. J. Cell Biol. 43:40-50. https://doi.org/10.1083/jcb.43.1.40

Nicklas, R.B., and C.A. Staehly. 1967. Chromosome micromanipulation. I. The mechanics of chromosome attachment to the spindle. Chromosoma. 21 1-16. https://doi.org/10.1007/BF00330544

Nicklas, R.B., D.F. Kubai, and T.S. Hays. 1982. Spindle microtubules and their mechanical associations after micromanipulation in anaphase. J. Cell Biol. 95:91-104. https://doi.org/10.1083/jcb.95.1.91

Nicklas, R.B., G.M. Lee, C.L. Rieder, and G. Rupp. 1989. Mechanically cut mitotic spindles: clean cuts and stable microtubules. J. Cell Sci. 94: $415-423$.

Oriola, D., D.J. Needleman, and J. Brugués. 2018. The Physics of the Metaphase Spindle. Annu. Rev. Biophys. 47:655-673. https://doi.org/10.1146/ annurev-biophys-060414-034107

Paliulis, L.V., and R.B. Nicklas. 2004. Micromanipulation of chromosomes reveals that cohesion release during cell division is gradual and does not require tension. Curr. Biol. 14:2124-2129. https://doi.org/10.1016/j.cub .2004 .11 .052

Portran, D., L. Schaedel, Z. Xu, M. Théry, and M.V. Nachury. 2017. Tubulin acetylation protects long-lived microtubules against mechanical ageing. Nat. Cell Biol. 19:391-398. https://doi.org/10.1038/ncb3481

Rieder, C.L.. 1981. The structure of the cold-stable kinetochore fiber in metaphase PtK1 cells. Chromosoma. 84:145-158. https://doi.org/10.1007/ BF00293368

Rieder, C.L., E.A. Davison, L.C.W. Jensen, L. Cassimeris, and E.D. Salmon. 1986. Oscillatory movements of monooriented chromosomes and their position relative to the spindle pole result from the ejection properties of the aster and half-spindle. J. Cell Biol. 103:581-591. https://doi.org/10 $.1083 /$ jcb.103.2.58

Sarangapani, K.K., and C.L. Asbury. 2014. Catch and release: how do kinetochores hook the right microtubules during mitosis? Trends Genet. 30 : 150-159. https://doi.org/10.1016/j.tig.2014.02.004

Sarangapani, K.K., B. Akiyoshi, N.M. Duggan, S. Biggins, and C.L. Asbury. 2013. Phosphoregulation promotes release of kinetochores from dynamic microtubules via multiple mechanisms. Proc. Natl. Acad. Sci. USA. 110:7282-7287. https://doi.org/10.1073/pnas.1220700110

Sasaki, T., N. Matsuki, and Y. Ikegaya. 2012. Targeted axon-attached recording with fluorescent patch-clamp pipettes in brain slices. Nat. Protoc. 7:1228-1234. https://doi.org/10.1038/nprot.2012.061

Schaedel, L., K. John, J. Gaillard, M.V. Nachury, L. Blanchoin, and M. Théry. 2015. Microtubules self-repair in response to mechanical stress. Nat. Mater. 14:1156-1163. https://doi.org/10.1038/nmat4396

Schindelin, J., I. Arganda-Carreras, E. Frise, V. Kaynig, M. Longair, T. Pietzsch, S. Preibisch, C. Rueden, S. Saalfeld, B. Schmid, et al. 2012. Fiji: an open-source platform for biological-image analysis. Nat. Methods. 9: 676-682. https://doi.org/10.1038/nmeth.2019

Shimamoto, Y., and T.M. Kapoor. 2012. Microneedle-based analysis of the micromechanics of the metaphase spindle assembled in Xenopus laevis egg extracts. Nat. Protoc. 7:959-969. https://doi.org/10.1038/nprot.2012 .033

Sikirzhytski, V., V. Magidson, J.B. Steinman, J. He, M. Le Berre, I. Tikhonenko, J.G. Ault, B.F. McEwen, J.K. Chen, H. Sui, et al. 2014. Di rect kinetochore-spindle pole connections are not required for chromosome segregation. J. Cell Biol. 206:231-243. https://doi.org/10 1083/jcb.201401090

Skibbens, R.V., and E.D. Salmon. 1997. Micromanipulation of chromosomes in mitotic vertebrate tissue cells: tension controls the state of kinetochore 
movement. Exp. Cell Res. 235:314-324. https://doi.org/10.1006/excr.1997 .3691

Skibbens, R.V., V.P. Skeen, and E.D. Salmon. 1993. Directional instability of kinetochore motility during chromosome congression and segregation in mitotic newt lung cells: a push-pull mechanism. J. Cell Biol. 122: 859-875. https://doi.org/10.1083/jcb.122.4.859

Skibbens, R.V., C.L. Rieder, and E.D. Salmon. 1995. Kinetochore motility after severing between sister centromeres using laser microsurgery: evidence that kinetochore directional instability and position is regulated by tension. J. Cell Sci. 108:2537-2548.

Spurck, T.P., O.G. Stonington, J.A. Snyder, J.D. Pickett-Heaps, A. Bajer, and J. Mole-Bajer. 1990. UV microbeam irradiations of the mitotic spindle. II. Spindle fiber dynamics and force production. J. Cell Biol. 111:1505-1518. https://doi.org/10.1083/jcb.111.4.1505

Suresh, P., A.F. Long, and S. Dumont. 2020. Microneedle manipulation of the mammalian spindle reveals specialized, short-lived reinforcement near chromosomes. eLife. 9. e53807. https://doi.org/10.7554/eLife.53807

Takagi, J., R. Sakamoto, G. Shiratsuchi, Y.T. Maeda, and Y. Shimamoto. 2019. Mechanically Distinct Microtubule Arrays Determine the Length and Force Response of the Meiotic Spindle. Dev. Cell. 49:267-278. https://do .org/10.1016/j.devcel.2019.03.014
Udy, D.B., M. Voorhies, P.P. Chan, T.M. Lowe, and S. Dumont. 2015. Draft De Novo Transcriptome of the Rat Kangaroo Potorous tridactylus as a Tool for Cell Biology. PLoS One. 10. e0134738. https://doi.org/10.1371/journal.pone.0134738

Vemu, A., E. Szczesna, E.A. Zehr, J.O. Spector, N. Grigorieff, A.M. Deaconescu, and A. Roll-Mecak. 2018. Severing enzymes amplify microtubule arrays through lattice GTP-tubulin incorporation. Science. 361: eaau1504.

Wan, X., D. Cimini, L.A. Cameron, and E.D. Salmon. 2012. The coupling between sister kinetochore directional instability and oscillations in centromere stretch in metaphase PtK1 cells. Mol. Biol. Cell. 23: 1035-1046. https://doi.org/10.1091/mbc.e11-09-0767

Ward, J.J., H. Roque, C. Antony, and F. Nédélec. 2014. Mechanical design principles of a mitotic spindle. eLife. 3. e03398. https://doi.org/10.7554/ eLife.03398

Yoo, T.Y., J.-M. Choi, W. Conway, C.-H. Yu, R.V. Pappu, and D.J. Needleman. 2018. Measuring NDC80 binding reveals the molecular basis of tensiondependent kinetochore-microtubule attachments. eLife. 7. e36392. https:// doi.org/10.7554/eLife.36392

Zhai, Y., P.J. Kronebusch, and G.G. Borisy. 1995. Kinetochore microtubule dynamics and the metaphase-anaphase transition. J. Cell Biol. 131: 721-734. https://doi.org/10.1083/jcb.131.3.721 


\section{Supplemental material}

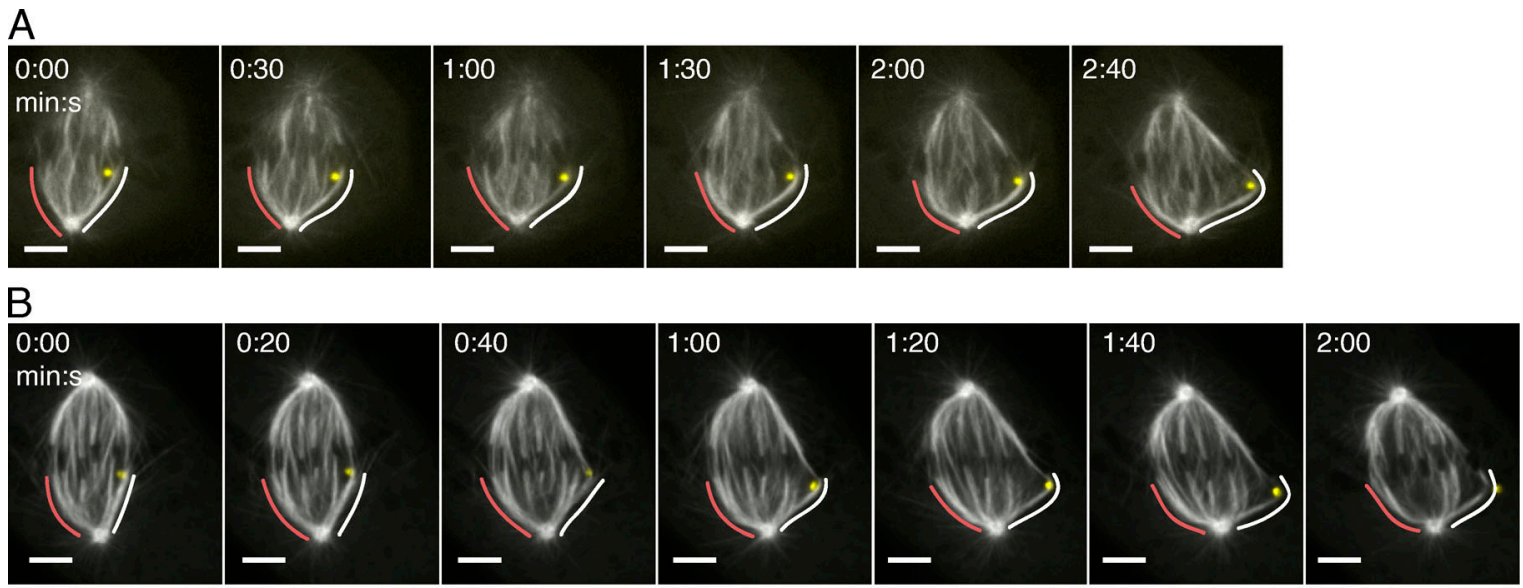

C

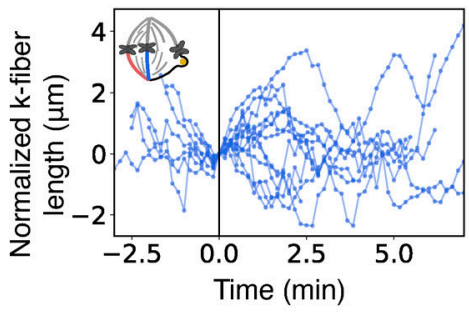

D

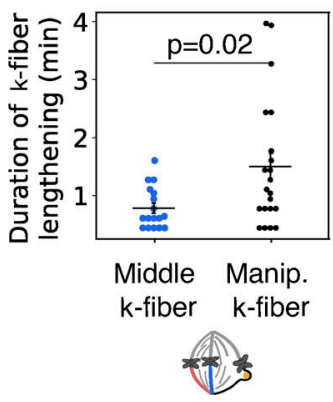

Figure S1. Individual k-fibers lengthen under applied force, related to Fig. 2. (A and B) Additional representative time-lapse images of spindle and k-fiber (GFP-tubulin, white) movement and remodeling in response to sustained force from a microneedle (Alexa 555, yellow) as in Fig. 2. (C) Plot of k-fiber length oscillations over time in neighboring middle $\mathrm{k}$-fibers (blue) during the manipulation of the neighboring outer k-fiber (black). (D) The average duration of the lengthening phase of the $k$-fiber is longer for the manipulated outer $k$-fibers (black; $n=21$ growth events in 18 cells) than for the unmanipulated neighboring middle $k$-fibers (blue; $n=17$ growth events in 13 cells; plot shows mean $\pm S E M, P=0.02$, two-sided Mann-Whitney $U$ test). Lengthening phases are scored as three or more consecutive time points with increasing $k$-fiber length.
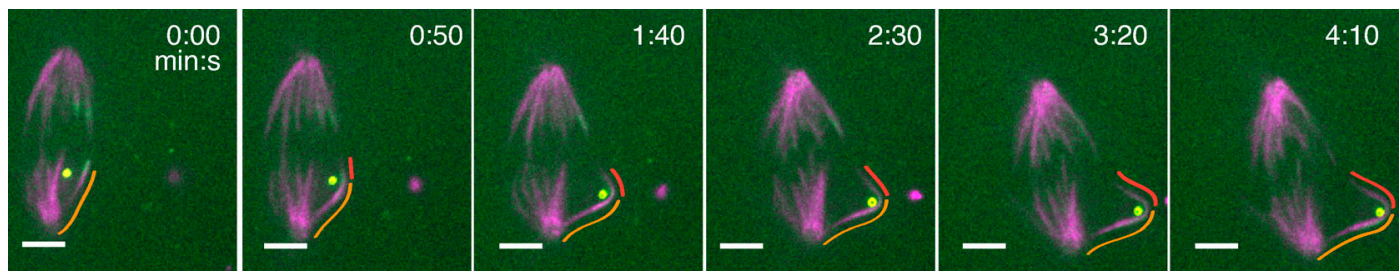

Figure S2. Force on individual mammalian k-fibers suppresses depolymerization at both ends, leading to net k-fiber lengthening, related to Fig. 3. Additional example time lapse of photomark (PA-GFP-tubulin, white) movement during microneedle (Alexa 555, yellow) manipulation of a k-fiber (SiR-tubulin, magenta). The distance between the photomark and the pole remains constant (orange line) while the distance between the photomark and plus end increases (red line). Scale bar, $4 \mu \mathrm{m}$. 
A

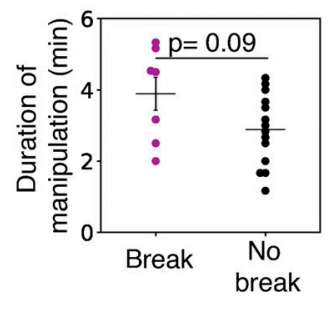

C
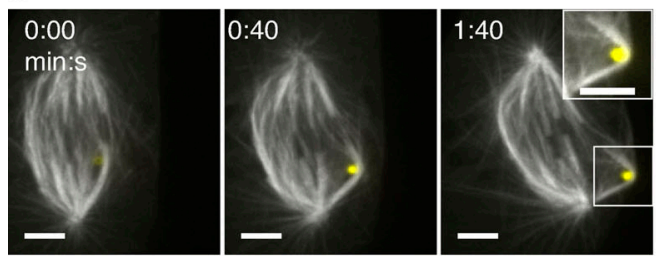
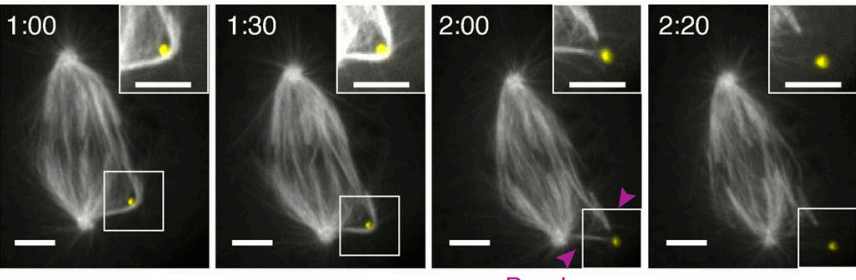

Break
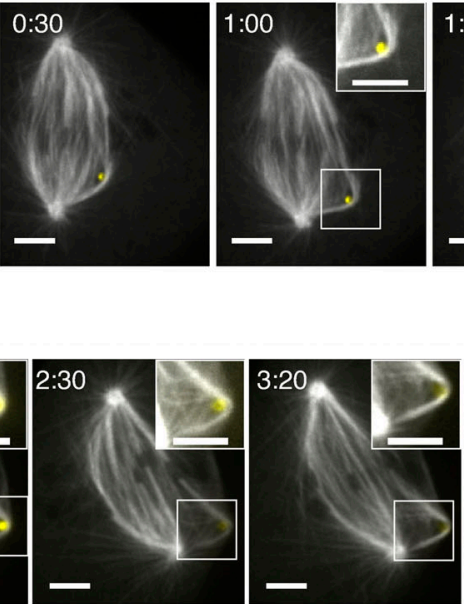
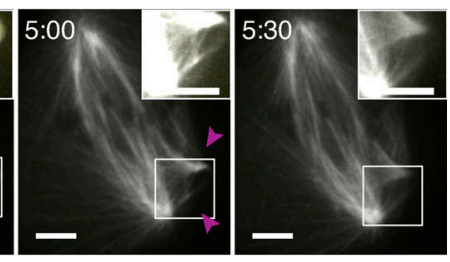

Break

Figure S3. Timescale of $\mathbf{k}$-fiber breakage under sustained force from microneedle, related to Fig. 4. (A) Plot of the duration of manipulation for cells whose $\mathrm{k}$-fibers broke or did not break under sustained applied force from the microneedle. The k-fibers broke when under force for slightly longer durations (plot shows mean $\pm \mathrm{SEM}, \mathrm{P}=0.09$, two-sided Mann-Whitney $U$ test). (B and C) Additional examples of time-lapse images of $k$-fiber (GFP-tubulin, white) bending, lengthening, and breaking under sustained force. Insets with enhanced contrast show local changes to the structure of the deformed $k$-fiber near the area of high curvature next to the microneedle (Alexa 647, yellow). The break creates two fragments of the original k-fiber (purple arrowheads). Scale bar, 4 $\mu \mathrm{m}$.

Video 1. Microneedle manipulation to exert sustained force on the mammalian k-fiber, related to Fig. 1. Microneedle manipulation of individual $k$-fiber in metaphase PtK2 cell to probe how k-fibers dynamics and structure respond to sustained force. The microneedle (Alexa 555, yellow) exerts force for minutes and moves the spindle (kinetochores, Cdc20-YFP, green; tubulin, SiR-tubulin, magenta) and deforms k-fibers. Manipulated spindles typically progress to anaphase (here at 10:04). Scale bar, $4 \mu \mathrm{m}$. Video was collected using a spinning disk confocal microscope at one frame every $4 \mathrm{~s}$. Video has been adjusted to play back at a constant rate of 30 frames/s. Video corresponds to still images from Fig. 1 D.

Video 2. The k-fibers persistently lengthen under applied force, related to Fig. 2. Microneedle manipulation of individual $k$-fiber in metaphase PtK2 cell results in $\mathrm{k}$-fiber (SiR-tubulin, white) lengthening and spindle translation and rotation in response to force. The microneedle (Alexa 555, yellow) exerts force for minutes starting at $\mathrm{t}=0$. Scale bar, $4 \mu \mathrm{m}$. Video was collected using a spinning disk confocal microscope at 1 frame every $10 \mathrm{~s}$. Video has been adjusted to play back at a constant rate of 15 frames/s. Video corresponds to still images from Fig. 2 B.

Video 3. The k-fiber lengthening under sustained force occurs by suppressing depolymerization at plus- and minus-ends, related to Fig. 3. Microneedle manipulation of individual k-fiber photomarked with PA-GFP-tubulin (white) in metaphase PtK1 cell reveals the mechanism of $k$-fiber lengthening under force. The microneedle (Alexa 555, yellow) exerts force on the k-fiber (SiR-tubulin, magenta) for minutes, and the photomark remains a constant distance from the pole but at a persistently increasing distance from the plus-end as the $k$-fiber lengthens, indicating a suppression of depolymerization at both ends. Scale bar, $4 \mu \mathrm{m}$. Video was collected using a spinning disk confocal microscope at one frame every $10 \mathrm{~s}$. Video has been adjusted to play back at a constant rate of 15 frames/s. Video corresponds to still images from Fig. 3 B.

Video 4. The k-fiber breakage occurs under sustained force for minutes, related to Fig. 4. Microneedle manipulation of individual $k$-fiber for minutes reveals k-fiber breakage instead of detachment from the kinetochore or pole. The microneedle (Alexa 555, yellow) exerts force on the k-fiber (GFP-tubulin, white) for minutes, and the k-fiber bends, lengthens, and ultimately breaks in two. Scale bar, $4 \mu \mathrm{m}$. Video was collected using a spinning disk confocal microscope at 1 frame every $10 \mathrm{~s}$. Video has been adjusted to play back at a constant rate of 15 frames/s. Video corresponds to still images from Fig. 4 B. 
Video 5. New k-fiber plus-ends can be stabilized after k-fiber breakage, related to Fig. 4. Microneedle manipulation of individual $k$-fiber reveals an example of stabilized bundle plus ends after $k$-fiber breakage. The microneedle (Alexa 555, yellow) exerts force on the k-fiber (SiR-tubulin, white) for minutes and is removed after the $k$-fiber breaks (purple arrowheads). The new plus end fragment of the bundle persists for minutes while the fragment attached to the kinetochore is reincorporated into the spindle. This video shows the later time points and response of the cell from Video 3 , where $t=0$ is the start of microneedle manipulation. Scale bar, $4 \mu \mathrm{m}$. Video was collected using a spinning disk confocal microscope at one frame every $10 \mathrm{~s}$. Video has been adjusted to play back at a constant rate of 15 frames/s. Video corresponds to still images from Fig. 4 G. 